\title{
Meteorology
}

\section{Multiple solutions, multi-site, and parameter transfer to calibrate DHSVM hydrological model}

\author{
Roberto Avelino Cecílio 1(1), Helder de Amorim Mendes "1(ㅇ, \\ Sidney Sara Zanetti ${ }^{1}$ (i) \\ ' Universidade Federal do Espírito Santo, Jerônimo Monteiro, ES, Brazil \\ "Universidade Federal do Espírito Santo, Alegre, ES, Brazil
}

\begin{abstract}
The application of hydrologic models often needs sets of input parameters related to environmental attributes which are not always available. This leads to the necessity of calibrating the input parameters. However, due to the non-linearity of the hydrologic phenomena, there may be multiple "best" solutions for the calibration. This paper proposes a method for calibrating the DHSVM hydrologic model using the concepts of multiple solutions, multi-site, and parameter transfer among catchments. Eight watersheds were calibrated, resulting in obtaining five sets of "best" parameters (clusters) for each one. Afterward, each watershed was modeled using the parameters of the other catchments in order to verify if the transfer of the calibrated parameters could promote satisfactory modeling of the streamflows. The results show that clusters calibrated for one watershed may be suitable for other catchments. Besdes, the calibrated parameters of the smaller catchments were satisfactory to simulate the streamflow of the bigger catchments. The proposed method can be useful in calibrating and extrapolating the input parameters to regions that do not have information about them
\end{abstract}

Keywords: Streamflow; Heuristics; Clustering search

\section{INTRODUCTION}

Hydrological modeling in a key factor to achieve sustainable management of the environment, especially concerning to integrated water resources management. Hydrological models are widely used computational tools to simulate hydrological responses of watersheds to human activities or meteorological variability. The elements that cause changes in the hydrological regime may be very diverse, such as 
changes in land use and management (HASSABALLAH et al., 2017; WOLDESENBET et al., 2017), adoption of best management practices (LIU et al., 2017; POTT; FOHRER, 2017), and the effects of climate change (FARJAD et al., 2017; NGUYEN et al., 2017).

Among the most complex hydrological models, the Distributed Hydrology Soil Vegetation Model (DHSVM) (WIGMOSTA; VAIL; LETTENMAIER, 1994) is a distributed model that divides the catchment into units in which simulations of climatic, vegetative and hydrological processes are performed through physical equations. The DHSVM has been successfully applied to simulate the hydrological behavior of watersheds in response to land use changes (ALVARENGA et al., 2016, 2017; CUARTAS et al., 2012; OLIVEIRA, 2014; THANAPAKPAWIN et al., 2007; WESTRICK; STORCK; MASS, 2002), and to climate change (BECKERS; SMERDON; WILSON, 2009; CUO; GIAMBELLUCA; ZIEGLER, 2011; SHOURONG et al., 2002; SUN et al., 2016; ZHANG et al., 2018).

The correct definition of the input parameters of a hydrological model, especially the DHSVM, is a key factor for hydrological modeling. Therefore, the success of hydrological modeling fundamentally depends on the quantification of the input parameter values of the model. However, a set of input parameters that provides good modeling is not always available (ARNOLD et al., 2015; MORIASI et al., 2015). When this information is not available, it is common to use data from literature or other studies of regions that have similar environmental characteristics (PINTO et al., 2013). However, although there is a similarity, the data does not always lead to satisfactory modeling (CECÍLIO et al., 2020).

In such situations, it is essential to perform the calibration of the input parameters in order to obtain adequate results for the modeling (DAGGUPATI et al., 2015; GUPTA; SOROOSHIAN; YAPO, 1999; YEN et al., 2015a). The calibration consists of varying the input parameter values of the model in order to obtain better adjustments in the modeling and can be performed manually or automatically (KAN et al., 2017). The manual process requires the researcher's experience and tends to be challenging (YEN et al., 2015a), time-consuming, boring, and tedious. For complex models that require a large number of input parameters, it is common for one or more parameters to 
influence the same model behavior, making the manual calibration process even more difficult. In this case, adjustment of the parameters becomes complex, since adjusting a given parameter may necessitate a new adjustment of a previously adjusted parameter (GUPTA; SOROOSHIAN; YAPO, 1999). The automatic process has become more common due to computational advances (ROUHOLAHNEJAD et al., 2012) with the application of several optimization techniques (KAN et al., 2017; TIGKAS; CHRISTELIS; TSAKIRIS, 2016) such as Genetic Algorithms (RESHMA et al., 2015; YAO; YANG, 2009) and Simulated Annealing (DAS et al., 2008; DATTA et al., 2017; SUMNER; MICK FLEMING; C. BATES, 1997), or even hybrid meta-heuristics (MENDES; CECÍLIO; ZANETTI, 2017).

However, due to several reasons, even automatic calibration can lead to unsatisfactory modeling. For example, the calibrated values may not be physically consistent. In addition, a calibration can be very useful for a given set of input data (meteorological, for example) or a specific basin. However, when the same calibration is applied to another set of input data, or even to a catchment upstream of the basin, the obtained results may not be satisfactory (GUPTA; SOROOSHIAN; YAPO, 1999; MELO NETO et al., 2014).

In order to minimize these problems, it is essential to find out and use new calibration techniques (ARSENAULT et al., 2014). As a premise of the present research, it is believed that the combination of three alternatives can provide better calibrations of hydrological models: existence of multiple optimal (or quasi-optimal) solutions (EASTON et al., 2007), multi-site calibration (BAI; SHEN; YAN, 2017; BRIGHENTI; BONUMÁ; CHAFFE, 2016) and the transfer of calibration parameters among catchments.

The calibration of distributed hydrologic models such as DHSVM is based on the solution of non-linear problems, which generally leads to the existence of multiple combinations of calibrated parameters that provide optimal or quasi-optimal solutions (DUAN; SOROOSHIAN; GUPTA, 1992; EASTON et al., 2007). In the context of the existence of multiple solutions for calibration, (MENDES; CECíLIO; ZANETTI, 2017) developed an automatic DHSVM calibrator based on the meta-heuristic Clustering Search (CS). This tool generates several different sets of optimal and quasi-optimal 
calibrated parameters, called clusters. One of the generated clusters holds the best solution obtained during the calibration process, while the others present other quasioptimal solutions.

For a large-scale watershed, single-site calibration and validation may ignore spatial heterogeneity and may not meet the needs of the entire watershed (BAI; SHEN; YAN, 2017). The multi-site method involves the division of the large watershed into smaller watersheds and applying the calibrated parameters of the multi-site calibration to the entire watershed (BAl; SHEN; YAN, 2017). Several authors have demonstrated the effectiveness of multi-site calibration over the single-site calibration centering solely on the data of the catchment outlet (BAl; SHEN; YAN, 2017; BRIGHENTI; BONUMÁ; CHAFFE, 2016; MOLINA-NAVARRO et al., 2017; SHRESTHA et al., 2016; WANG et al., 2012).

It is imperative to investigate the feasibility of not only using the calibrated parameters that provide the best solution for a basin, but also those that provide quasioptimal solutions. Moreover, it is feasible that such a procedure can be done for more than one catchment within the same basin. Besides, it is subsequently possible to transfer the different sets of calibrated parameters (optimal and quasi-optimal) to each catchment and to verify the efficiency of each set in the modeling of all the others. Therefore, the objective of this paper was to propose a method for calibrating the DHSVM hydrologic model using the concepts of multiple solutions, multi-site, and parameter transfer among catchments.

\section{MATERIAL AND METHODS}

A computational tool was implemented to calibrate many sets of input parameters to several watersheds of a specific basin. The sets of calibrated input parameters were then applied to all the watersheds intending to verify the DHSVM accuracy. The implemented steps and data are summarized in the flowchart in Figure1. 
Figure 1 - Flowchart of the steps accomplished in this paper

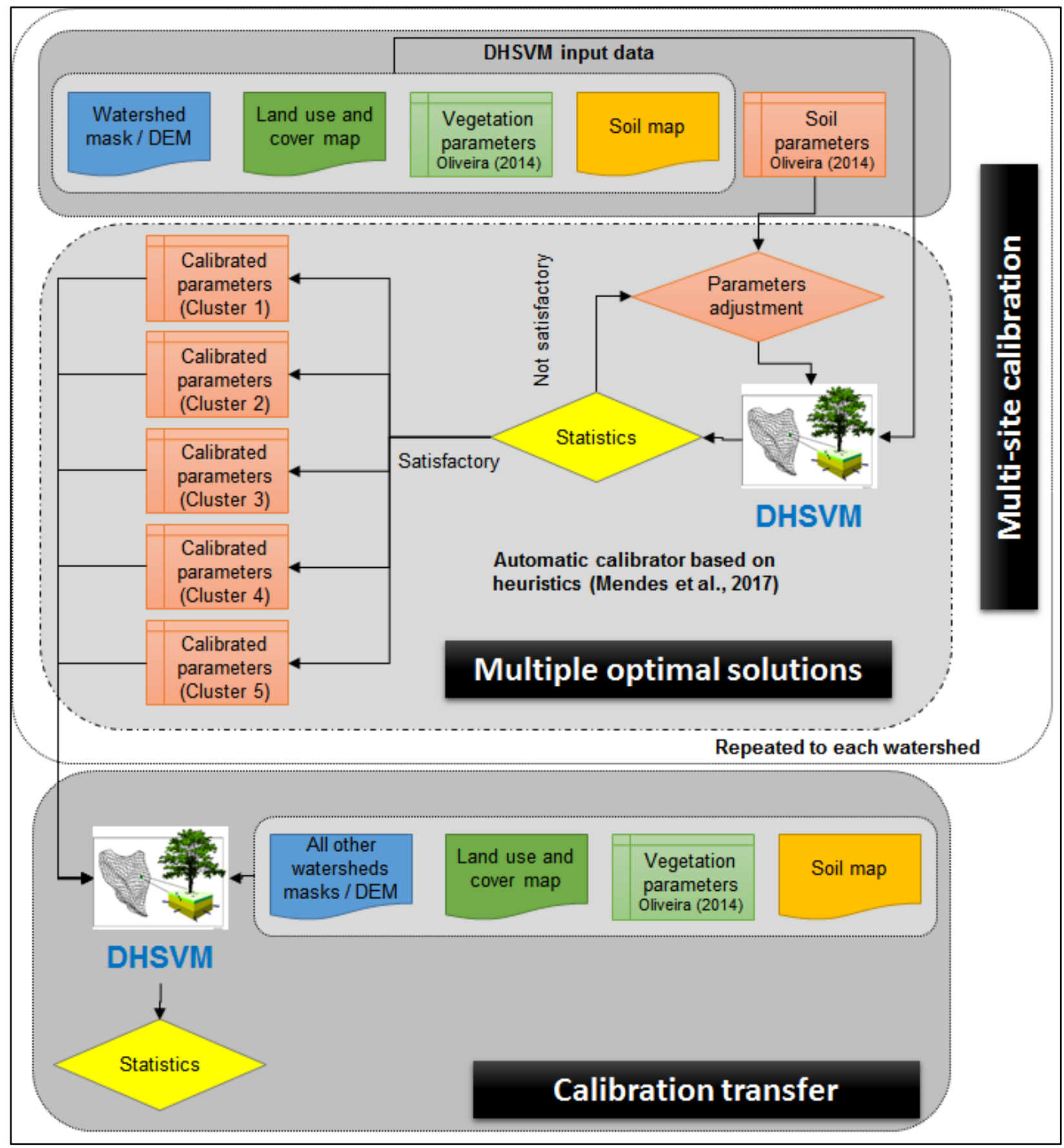

\subsection{Basin application}

The areas understudy were the Itapemirim River Basin (IRB), and its watersheds (sub-basins) named Castelo, Fazenda Lajinha, Itaici, lúna, Rive, Terra Corrida, Usina Paineiras, and Usina São Miguel (Figure 2). The information regarding the fluviometric stations at the mouth of each watershed is presented in 
Table 1. The IRB is located in the southern region of Espírito Santo State (Figure 2), with an area of $6,014 \mathrm{~km}^{2}$. According to the Köppen classification, the predominant climatic types of the regions are "Cwa" - Humid Subtropical climate with dry winter and hot summer, and "Cwb" - Humid Subtropical climate with a dry winter and temperate summer (ALVARES et al., 2013). The vegetation is within the domains of the Atlantic Forest Biome with the following formations: Dense Ombrophilous Forest and Semi-deciduous Seasonal Forest (INSTITUTO BRASILEIRO DE GEOGRAFIA E ESTATÍSTICA, 2012). The relief is dominated by mountains, with higher elevations in the proximities of the western contour, which indicates suitability for applying the DHSVM (BECKERS; SMERDON; WILSON, 2009; SURFLEET; SKAUGSET; MCDONNELL, 2010).

Figure 2 - Geographical location of the IRB and stream gauge stations

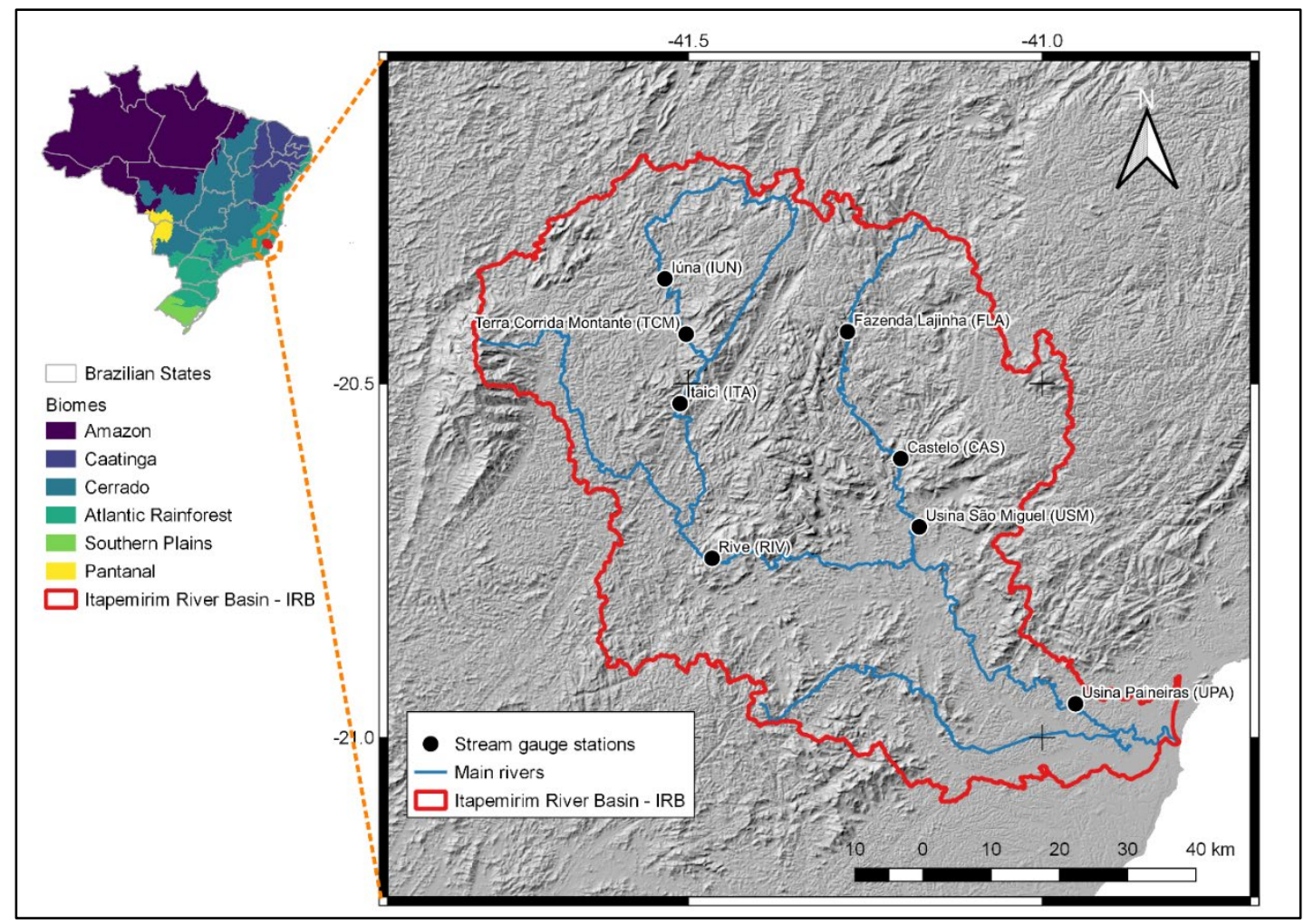


Table 1 - Stream gauge station locations and its watershed areas

\begin{tabular}{lcccc}
\hline Station & Latitude & Longitude & Altitude (m) & Drainage area $\mathbf{( K m}^{2}$ ) \\
\hline Fazenda Lajinha (FLA) & $-20^{\circ} 25^{\prime}$ & $-41^{\circ} 16^{\prime}$ & 400 & 436 \\
lúna (IUN) & $-20^{\circ} 21^{\prime}$ & $-41^{\circ} 31^{\prime}$ & 640 & 432 \\
Terra Corrida (TCO) & $-20^{\circ} 25^{\prime}$ & $-41^{\circ} 30^{\prime}$ & 380 & 587 \\
Castelo (CAS) & $-20^{\circ} 36^{\prime}$ & $-41^{\circ} 11^{\prime}$ & 107 & 975 \\
Itaici (ITA) & $-20^{\circ} 31^{\prime}$ & $-41^{\circ} 30^{\prime}$ & 380 & 1045 \\
Usina São Miguel (USM) & $-20^{\circ} 42^{\prime}$ & $-41^{\circ} 10^{\prime}$ & 200 & 1459 \\
Rive (RIV) & $-20^{\circ} 44^{\prime}$ & $-41^{\circ} 27^{\prime}$ & 128 & 2218 \\
Usina Paineiras (UPA) & $-20^{\circ} 57^{\prime}$ & $-41^{\circ} 57^{\prime}$ & 40 & 5168 \\
\hline
\end{tabular}

\subsection{DHSVM database}

DHSVM is a physically based distributed hydrologic model that explicitly solves water and energy balances for each model grid cell. It accounts for the effects of climate, topography and land cover by explicitly representing the spatial distribution of soil properties, soil depth, vegetation properties, and elevation. DHSVM incorporates canopy precipitation interception, evapotranspiration, energy, and radiation balance, snow accumulation and melt, runoff generation through saturation excess and infiltration excess mechanisms, unsaturated soil moisture movement, saturated subsurface flow, and groundwater recharge and discharge (CUO et al., 2006). Briefly described, DHSVM calculates the spatial distribution of soil the moisture and hydrological elements (evapotranspiration, runoff, and streamflow) in time increments for each individual grid cells of the digital elevation model of the watershed. The water balance is calculated for each grid cell based on effects from vegetation, meteorological elements, soil properties, and relief. The model uses a two-layer canopy representation to calculate interception and evapotranspiration, a multilayer unsaturated soil model based on Darcy's Law, and a saturated subsurface flow model. Once the model completes the water balance calculations, each grid cell exchanges water with the adjacent grid cells, which results in a three-dimensional redistribution of surface and subsurface water across the watershed (SURFLEET; SKAUGSET; MCDONNELL, 2010). The multi-layer soil column in each pixel is a 
series of soil moisture reservoirs, and saturated subsurface flow exists in the deepest soil layer. Saturation excess and infiltration excess mechanisms represent runoff generation. Stream segment storage volume is computed using linear-reservoir routing (THANAPAKPAWIN et al., 2007). A detailed description of DHSVM can be found in (WIGMOSTA et al., 2002; WIGMOSTA; VAIL; LETTENMAIER, 1994).

Model parameters fall under meteorological, soil, vegetation (land cover), and topography categories. The model's spatial framework is set by a digital elevation model (DEM) of user-defined resolution (pixel size). The DEM source was the Global Digital Elevation Model (GDEM2) from the Advanced Spaceborne Thermal Emission and Reflection Radiometer (ASTER), with the spatial resolution previously established in 100 m (MENDES, 2016).

The daily meteorological information was obtained from meteorological and rain gauge stations inside and surrounding the IRB (Figure 3), accomplishing the period from 2007 to 2011 (same period of the daily streamflow data).

Figure 3 - Geographical location of the meteorological and rain gauge stations

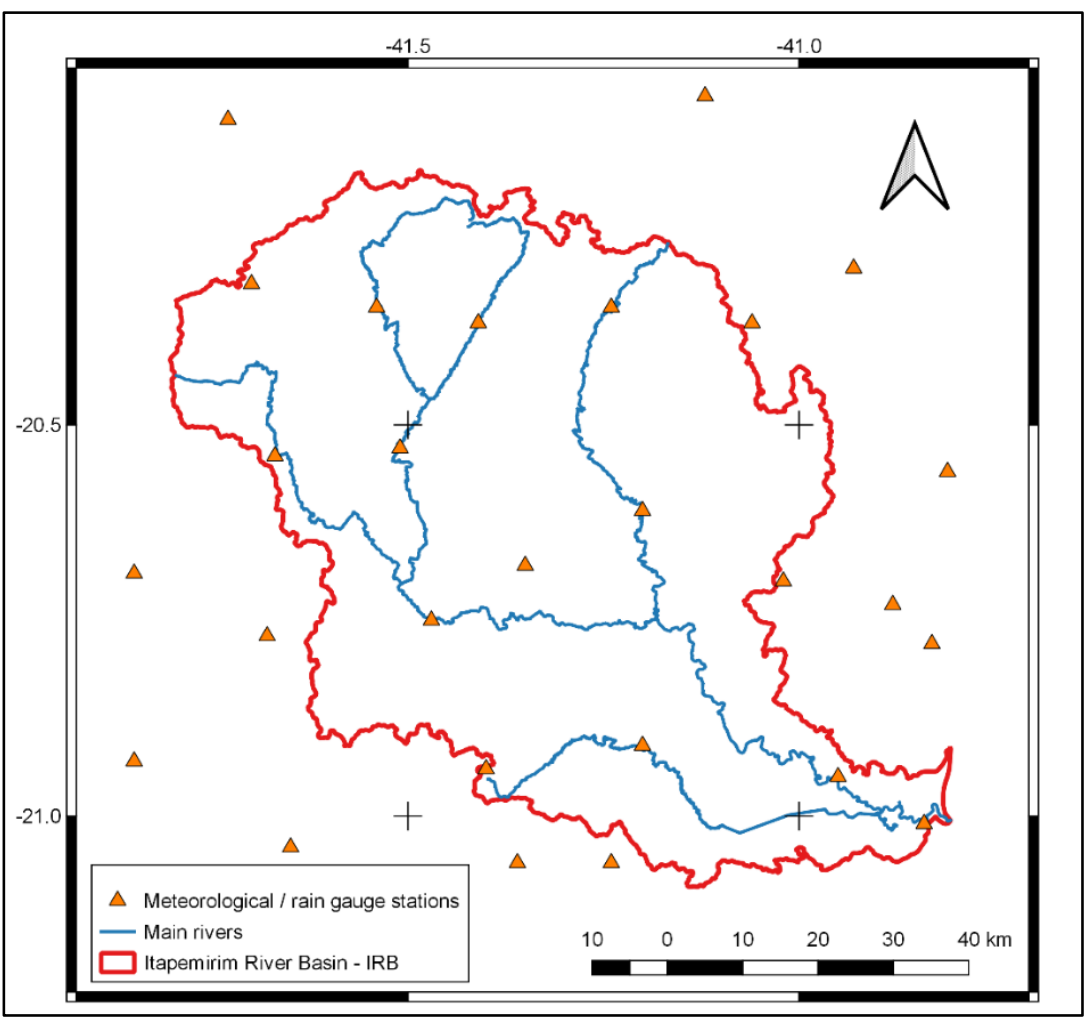


Soil data and maps (Figure 4a) were obtained from the Brazilian Soils Map in the scale 1: 5,000,000 (EMBRAPA - EMPRESA BRASILEIRA DE PESQUISA AGROPECUÁRIA, 2013; SANTOS et al., 2011), showing the occurrence of Oxisols, Interceptisols, and Ultisols. The land use map (Figure 4b) was obtained from the Espírito Santo State Environmental Institute (GEOBASES, 2012). There are eight main land use classes: pasture, agricultural crops, buildings, planted forests (pinus and eucalyptus), swamp, native forest, water bodies, and rocks.

Figure 4 - Soil (a) and land use (b) maps of the Itapemirim River Basin

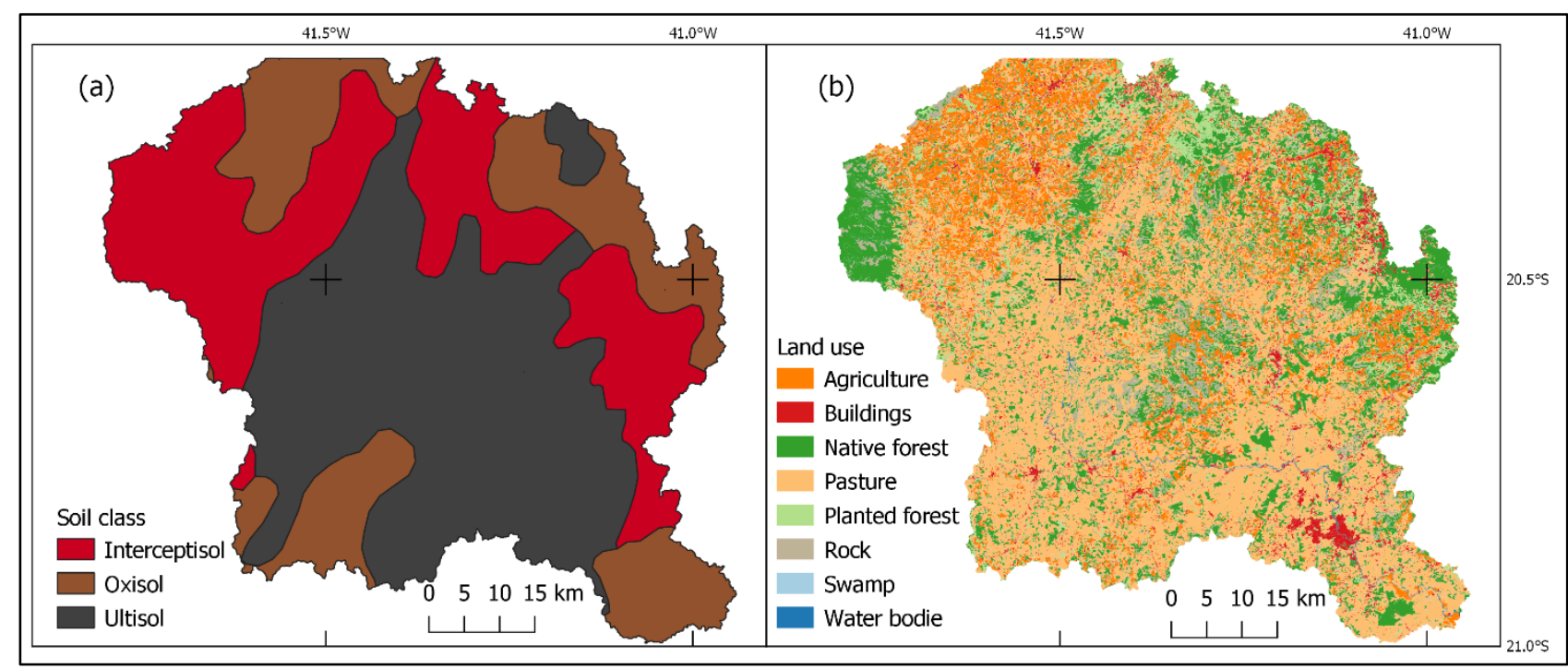

There is currently a lack of information on the soil and vegetation parameters required for DHSVM application in Brazil, especially in the Atlantic Forest. Thus, in order to obtain the soil parameters, data were collected from previous studies about DHSVM application in Brazil (KRUK et al., 2009; OLIVEIRA, 2014). Oliveira (2014) carried out modeling of the Jucu River Basin located close to the IRB, and is relatively similar in soil, climate and land use characteristics. Thus, vegetation and soil initial parameters, except maximum soil depth, were taken from this study. Soil maximum depth was set in $30 \mathrm{~m}$, according to a previous IRB study (MENDES, 2016). 


\subsection{Calibration of input parameters to each watershed}

Each of the eight watersheds was calibrated using a tool based on CS heuristics (MENDES; CECÍLIO; ZANETTI, 2017). The calibrated parameters were those presented in Table 2 and the calibrator was set to save five different "best" solutions (clusters). Thus, the five "best" calibrated parameter sets were obtained for each watershed at the end of the calibration, totaling 40 solution files.

Table 2 - Soil input parameters taken at DHSVM calibration (KIs is the Lateral saturated hydraulic conductivity; EDR is the Exponential decrease rate of lateral saturated hydraulic conductivity; MIC is the Maximum infiltration capacity; and P is the Porosity of the soil layers)

\begin{tabular}{|c|c|c|c|c|}
\hline Soil & Input parameter & Initial value & Lower value & Upper value \\
\hline \multirow{6}{*}{ Interceptisol } & $\mathrm{Kls}\left(\mathrm{x} 10^{-5} \mathrm{~m} \mathrm{~s}^{-1}\right)$ & 2.17 & 1.00 & 9.00 \\
\hline & EDR (dimensionless) & 0.23 & 0.05 & 0.50 \\
\hline & $\operatorname{MIC}\left(\times 10^{-7} \mathrm{~m} \mathrm{~s}^{-1}\right)$ & 4.0 & 3.0 & 9.0 \\
\hline & $P\left(m^{3} m^{-3}\right)-1 s t$ layer & 0.52 & 0.31 & 0.60 \\
\hline & $P\left(m^{3} m^{-3}\right)-2$ nd layer & 0.54 & 0.33 & 0.60 \\
\hline & $P\left(m^{3} m^{-3}\right)-3 r d$ layer & 0.55 & 0.31 & 0.60 \\
\hline \multirow{6}{*}{ Oxisol } & $\mathrm{Kls}\left(\mathrm{x} 10^{-5} \mathrm{~m} \mathrm{~s}^{-1}\right)$ & 2.71 & 1.00 & 9.00 \\
\hline & EDR (dimensionless) & 0.14 & 0.05 & 0.50 \\
\hline & $\operatorname{MIC}\left(\times 10^{-7} \mathrm{~m} \mathrm{~s}^{-1}\right)$ & 5.0 & 3.0 & 9.0 \\
\hline & $P\left(m^{3} m^{-3}\right)-1 s t$ layer & 0.54 & 0.31 & 0.60 \\
\hline & $P\left(m^{3} m^{-3}\right)-2$ nd layer & 0.53 & 0.31 & 0.60 \\
\hline & $P\left(m^{3} m^{-3}\right)-3 r d$ layer & 0.51 & 0.31 & 0.60 \\
\hline \multirow{6}{*}{ Ultisol } & $\operatorname{Kls}\left(\times 10^{-5} \mathrm{~m} \mathrm{~s}^{-1}\right)$ & 2.46 & 1.00 & 9.00 \\
\hline & EDR (dimensionless) & 0.33 & 0.05 & 0.50 \\
\hline & $\operatorname{MIC}\left(\times 10^{-7} \mathrm{~m} \mathrm{~s}^{-1}\right)$ & 5.0 & 3.0 & 9.0 \\
\hline & $P\left(m^{3} m^{-3}\right)-1 s t$ layer & 0.48 & 0.31 & 0.60 \\
\hline & $P\left(m^{3} m^{-3}\right)-2$ nd layer & 0.47 & 0.31 & 0.60 \\
\hline & $P\left(m^{3} m^{-3}\right)-3 r d$ layer & 0.47 & 0.31 & 0.60 \\
\hline
\end{tabular}


The calibration was set to maximize Nash-Sutcliffe efficiency (NS) (NASH; SUTCLIFFE, 1970) and minimize the percentage of bias (PBIAS) (MORIASI et al., 2007), presented in equations 1 and 2, respectively:

$$
\begin{aligned}
& \text { NS }=1-\frac{\sum_{i=1}^{n}\left(Q_{i}^{\text {obs }}-Q_{i}^{\text {sim }}\right)^{2}}{\sum_{i=1}^{n}\left(Q_{i}^{\text {obs }}-Q_{\operatorname{man}}^{\text {obs }}\right)^{2}} \\
& \text { PBLAS }=100 \frac{\sum_{i=1}^{n}\left(Q_{i}^{o b s}-Q_{i}^{\text {sim }}\right)}{\sum_{i=1}^{n}\left(Q_{i}^{\text {obs }}\right)}
\end{aligned}
$$

where $\mathrm{Q}^{\text {obs }} \mathrm{i}$ is the observed streamflow; $\mathrm{Q}^{\text {sim }} \mathrm{i}$ is the simulated streamflow; $\mathrm{n}$ is the number of observations; and $\mathrm{Q}_{\text {mean }}^{\text {obs }}$ is the average observed streamflow.

The efficiency of the modeling on a daily basis was taken as satisfactory when NS $\geq 0.30$ (NOORI; KALIN, 2016) and $-25 \% \leq$ BIAS $\leq 25 \%$ (MORIASI et al., 2007).

\subsection{Transfer of calibrated parameters sets among watersheds}

The transfer of the solutions obtained with each calibration was performed for all watersheds. DHSVM was applied to each watershed using all the 35 solutions (set of parameters) obtained for the other seven watersheds. The NS and PBIAS of each transfer were also calculated.

\section{RESULTS}

The results are presented considering three groups of watersheds: Group I, upstream of the Rive station (IUN, TCO, ITA, and RIV); Group II, upstream of the Usina São Miguel station (FLA, CAS, and USM); and Group III, the only station further downstream of IRB (UPA). 


\subsection{Calibration of each watershed}

Tables 3 to 5 present the five best sets of calibrated parameters (clusters) for each watershed, and its respective NS and PBIAS indexes.

Table 3 - Calibrated parameters and performance indexes for the five clusters of each watershed upstream from RIV station

\begin{tabular}{|c|c|c|c|c|c|c|c|}
\hline Basin & Cluster & Parameter & Ultisol & Oxisol & Interceptisol & NS & PBIAS (\%) \\
\hline \multirow{30}{*}{ IUN } & \multirow{6}{*}{ IUN10 } & $\mathrm{K}_{\mathrm{Is}}\left(\mathrm{x} 10^{-5} \mathrm{~m} \mathrm{~s}^{-1}\right)$ & - & 5.11 & 4.57 & \multirow{6}{*}{0.41} & \multirow{6}{*}{15} \\
\hline & & EDR (dimensionless) & - & 0.34 & 0.43 & & \\
\hline & & $\operatorname{MIC}\left(x 10^{-7} \mathrm{~m} \mathrm{~s}^{-1}\right)$ & - & 8.47 & 8.63 & & \\
\hline & & $P\left(m^{3} m^{-3}\right)-1 s t$ layer & - & 0.59 & 0.47 & & \\
\hline & & $P\left(m^{3} m^{-3}\right)-2 n d$ layer & - & 0.58 & 0.59 & & \\
\hline & & $P\left(m^{3} m^{-3}\right)-3 r d$ layer & - & 0.46 & 0.60 & & \\
\hline & \multirow{6}{*}{ IUN2 } & $\mathrm{K}_{\mathrm{Is}}\left(\times 10^{-5} \mathrm{~m} \mathrm{~s}^{-1}\right)$ & - & 4.81 & 4.87 & \multirow{6}{*}{0.39} & \multirow{6}{*}{17} \\
\hline & & EDR (dimensionless) & - & 0.34 & 0.33 & & \\
\hline & & $\operatorname{MIC}\left(x 10^{-7} \mathrm{~m} \mathrm{~s}^{-1}\right)$ & - & 8.47 & 8.63 & & \\
\hline & & $P\left(m^{3} m^{-3}\right)-1 s t$ layer & - & 0.54 & 0.42 & & \\
\hline & & $P\left(m^{3} m^{-3}\right)-2 n d$ layer & - & 0.58 & 0.59 & & \\
\hline & & $P\left(m^{3} m^{-3}\right)-3 r d$ layer & - & 0.46 & 0.60 & & \\
\hline & \multirow{6}{*}{ IUN3 } & $\mathrm{K}_{\mathrm{ls}}\left(\times 10^{-5} \mathrm{~m} \mathrm{~s}^{-1}\right)$ & - & 4.81 & 4.57 & \multirow{6}{*}{0.38} & \multirow{6}{*}{18} \\
\hline & & EDR (dimensionless) & - & 0.34 & 0.23 & & \\
\hline & & $\operatorname{MIC}\left(x 10^{-7} \mathrm{~m} \mathrm{~s}^{-1}\right)$ & - & 8.47 & 8.63 & & \\
\hline & & $P\left(m^{3} m^{-3}\right)-1$ st layer & - & 0.59 & 0.42 & & \\
\hline & & $P\left(m^{3} m^{-3}\right)-2 n d$ layer & - & 0.58 & 0.59 & & \\
\hline & & $P\left(m^{3} m^{-3}\right)-3 r d$ layer & - & 0.46 & 0.60 & & \\
\hline & \multirow{6}{*}{ IUN4 } & $\mathrm{K}_{\mathrm{ls}}\left(\times 10^{-5} \mathrm{~m} \mathrm{~s}^{-1}\right)$ & - & 4.81 & 4.27 & \multirow{6}{*}{0.38} & \multirow{6}{*}{18} \\
\hline & & EDR (dimensionless) & - & 0.34 & 0.23 & & \\
\hline & & $\operatorname{MIC}\left(\times 10^{-7} \mathrm{~m} \mathrm{~s}^{-1}\right)$ & - & 8.47 & 8.63 & & \\
\hline & & $P\left(m^{3} m-3\right)-1$ st layer & - & 0.59 & 0.42 & & \\
\hline & & $P\left(m^{3} m^{-3}\right)-2 n d$ layer & - & 0.58 & 0.59 & & \\
\hline & & $P\left(m^{3} m^{-3}\right)-3 r d$ layer & - & 0.46 & 0.60 & & \\
\hline & \multirow{6}{*}{ IUN5 } & $\mathrm{K}_{\mathrm{Is}}\left(\times 10^{-5} \mathrm{~m} \mathrm{~s}^{-1}\right)$ & - & 3.91 & 4.27 & \multirow{6}{*}{0.49} & \multirow{6}{*}{9} \\
\hline & & EDR (dimensionless) & - & 0.34 & 0.43 & & \\
\hline & & $\operatorname{MIC}\left(\times 10^{-7} \mathrm{~m} \mathrm{~s}^{-1}\right)$ & - & 8.47 & 8.63 & & \\
\hline & & $P\left(m^{3} m^{-3}\right)-1$ st layer & - & 0.59 & 0.47 & & \\
\hline & & $P\left(m^{3} m^{-3}\right)-2 n d$ layer & - & 0.58 & 0.54 & & \\
\hline & & $P\left(m^{3} m^{-3}\right)-3 r d$ layer & - & 0.46 & 0.45 & & \\
\hline
\end{tabular}


Table 3 - Continuation...

\begin{tabular}{|c|c|c|c|c|c|c|c|}
\hline Basin & Cluster & Parameter & Ultisol & Oxisol & Interceptisol & NS & PBIAS (\%) \\
\hline \multirow{30}{*}{ TCO } & \multirow{6}{*}{ TCO1 } & $\mathrm{K}_{\mathrm{Is}}\left(\mathrm{x} 10^{-5} \mathrm{~m} \mathrm{~s}^{-1}\right)$ & - & 8.71 & 3.36 & \multirow{6}{*}{0.54} & \multirow{6}{*}{ 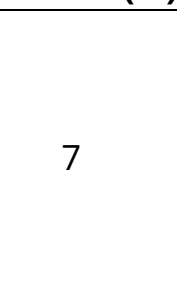 } \\
\hline & & EDR (dimensionless) & - & 0.44 & 0.33 & & \\
\hline & & $\operatorname{MIC}\left(\times 10^{-7} \mathrm{~m} \mathrm{~s}^{-1}\right)$ & - & 8.47 & 8.63 & & \\
\hline & & $P\left(m^{3} m^{-3}\right)-1$ st layer & - & 0.59 & 0.58 & & \\
\hline & & $P\left(m^{3} m^{-3}\right)-2 n d$ layer & - & 0.48 & 0.57 & & \\
\hline & & $P\left(m^{3} m^{-3}\right)-3 r d$ layer & - & 0.56 & 0.42 & & \\
\hline & \multirow{6}{*}{$\mathrm{TCO} 2$} & $\mathrm{~K}_{\mathrm{ls}}\left(\times 10^{-5} \mathrm{~m} \mathrm{~s}^{-1}\right)$ & - & 8.71 & 4.26 & \multirow{6}{*}{0.53} & \multirow{6}{*}{13} \\
\hline & & EDR (dimensionless) & - & 0.24 & 0.43 & & \\
\hline & & $\operatorname{MIC}\left(x 10^{-7} \mathrm{~m} \mathrm{~s}^{-1}\right)$ & - & 8.47 & 8.63 & & \\
\hline & & $P\left(m^{3} m-{ }^{3}\right)-1$ st layer & - & 0.54 & 0.58 & & \\
\hline & & $P\left(m^{3} m^{-3}\right)-2 n d$ layer & - & 0.58 & 0.57 & & \\
\hline & & $P\left(m^{3} m^{-3}\right)-3 r d$ layer & - & 0.56 & 0.42 & & \\
\hline & \multirow{6}{*}{ TCO3 } & $\mathrm{K}_{\mathrm{Is}}\left(\times 10^{-5} \mathrm{~m} \mathrm{~s}^{-1}\right)$ & - & 8.71 & 3.96 & \multirow{6}{*}{0.50} & \multirow{6}{*}{9} \\
\hline & & EDR (dimensionless) & - & 0.34 & 0.43 & & \\
\hline & & $\operatorname{MIC}\left(x 10^{-7} \mathrm{~m} \mathrm{~s}^{-1}\right)$ & - & 8.47 & 8.63 & & \\
\hline & & $P\left(m^{3} m^{-3}\right)-1 s t$ layer & - & 0.54 & 0.58 & & \\
\hline & & $P\left(m^{3} m^{-3}\right)-2 n d$ layer & - & 0.48 & 0.57 & & \\
\hline & & $P\left(m^{3} m^{-3}\right)-3 r d$ layer & - & 0.56 & 0.47 & & \\
\hline & \multirow{6}{*}{$\mathrm{TCO} 4$} & $\mathrm{~K}_{\mathrm{Is}}\left(\times 10^{-5} \mathrm{~m} \mathrm{~s}^{-1}\right)$ & - & 8.71 & 3.36 & \multirow{6}{*}{0.54} & \multirow{6}{*}{7} \\
\hline & & EDR (dimensionless) & - & 0.44 & 0.43 & & \\
\hline & & $\operatorname{MIC}\left(x 10^{-7} \mathrm{~m} \mathrm{~s}^{-1}\right)$ & - & 8.47 & 8.63 & & \\
\hline & & $P\left(m^{3} m^{-3}\right)-1$ st layer & - & 0.54 & 0.58 & & \\
\hline & & $P\left(m^{3} m^{-3}\right)-2 n d$ layer & - & 0.53 & 0.57 & & \\
\hline & & $P\left(m^{3} m^{-3}\right)-3 r d$ layer & - & 0.56 & 0.42 & & \\
\hline & \multirow{6}{*}{ TCO5 } & $\mathrm{K}_{\mathrm{ls}}\left(\times 10^{-5} \mathrm{~m} \mathrm{~s}^{-1}\right)$ & - & 8.71 & 3.36 & \multirow{6}{*}{0.54} & \multirow{6}{*}{7} \\
\hline & & EDR (dimensionless) & - & 0.44 & 0.43 & & \\
\hline & & $\operatorname{MIC}\left(x 10^{-7} \mathrm{~m} \mathrm{~s}^{-1}\right)$ & - & 8.47 & 8.63 & & \\
\hline & & $P\left(m^{3} m^{-3}\right)$ - 1st layer & - & 0.59 & 0.58 & & \\
\hline & & $P\left(m^{3} m^{-3}\right)-2 n d$ layer & - & 0.48 & 0.57 & & \\
\hline & & $P\left(m^{3} m^{-3}\right)-3 r d$ layer & - & 0.56 & 0.42 & & \\
\hline \multirow{12}{*}{ ITA } & \multirow{6}{*}{ ITA1 } & $\mathrm{K}_{\mathrm{ls}}\left(\times 10^{-5} \mathrm{~m} \mathrm{~s}^{-1}\right)$ & 2.76 & $5 . .11$ & $1 . .57$ & \multirow{6}{*}{0.49} & \multirow{6}{*}{6} \\
\hline & & EDR (dimensionless) & 0.43 & $0 . .14$ & $0 . .43$ & & \\
\hline & & $\operatorname{MIC}\left(x 10^{-7} \mathrm{~m} \mathrm{~s}^{-1}\right)$ & 6.74 & $8 . .47$ & $8 . .63$ & & \\
\hline & & $P\left(m^{3} m^{-3}\right)-1$ st layer & 0.52 & $0 . .59$ & $0 . .52$ & & \\
\hline & & $P\left(m^{3} m^{-3}\right)-2 n d$ layer & 0.59 & $0 . .48$ & $0 . .54$ & & \\
\hline & & $P\left(m^{3} m^{-3}\right)-3 r d$ layer & 0.48 & $0 . .56$ & $0 . .55$ & & \\
\hline & \multirow{6}{*}{ ITA2 } & $\mathrm{K}_{\mathrm{Is}}\left(\times 10^{-5} \mathrm{~m} \mathrm{~s}^{-1}\right)$ & 3.36 & $5 . .11$ & $2 . .17$ & \multirow{6}{*}{0.44} & \multirow{6}{*}{9} \\
\hline & & EDR (dimensionless) & 0.33 & $0 . .14$ & $0 . .43$ & & \\
\hline & & $\operatorname{MIC}\left(x 10^{-7} \mathrm{~m} \mathrm{~s}^{-1}\right)$ & 7.90 & 8.47 & 8.63 & & \\
\hline & & $P\left(m^{3} m^{-3}\right)-1$ st layer & 0.47 & 0.59 & 0.52 & & \\
\hline & & $P\left(m^{3} m^{-3}\right)-2 n d$ layer & 0.54 & 0.48 & 0.59 & & \\
\hline & & $P\left(m^{3} m^{-3}\right)-3 r d$ layer & 0.48 & 0.56 & 0.60 & & \\
\hline
\end{tabular}

Continuation... 


\begin{tabular}{|c|c|c|c|c|c|c|c|}
\hline Basin & Cluster & Parameter & Ultisol & Oxisol & Interceptisol & NS & PBIAS (\%) \\
\hline & \multirow{6}{*}{ ITA3 } & $\mathrm{K}_{\mathrm{Is}}\left(\times 10^{-5} \mathrm{~m} \mathrm{~s}^{-1}\right)$ & 3.06 & 5.11 & 2.17 & \multirow{6}{*}{0.45} & \multirow{6}{*}{9} \\
\hline & & EDR (dimensionless) & 0.33 & 0.14 & 0.43 & & \\
\hline & & $\operatorname{MIC}\left(\times 10^{-7} \mathrm{~m} \mathrm{~s}^{-1}\right)$ & 7.32 & 8.47 & 8.63 & & \\
\hline & & $P\left(m^{3} m^{-3}\right)-1 s t$ layer & 0.52 & 0.59 & 0.52 & & \\
\hline & & $P\left(m^{3} m^{-3}\right)-2$ nd layer & 0.54 & 0.48 & 0.59 & & \\
\hline & & $P\left(m^{3} m^{-3}\right)$ - 3rd layer & 0.43 & 0.56 & 0.60 & & \\
\hline & \multirow{6}{*}{ ITA4 } & $\mathrm{K}_{\mathrm{Is}}\left(\mathrm{x} 10^{-5} \mathrm{~m} \mathrm{~s}^{-1}\right)$ & 3.06 & 5.11 & 2.17 & \multirow{6}{*}{0.46} & \multirow{6}{*}{9} \\
\hline & & EDR (dimensionless) & 0.33 & 0.14 & 0.43 & & \\
\hline & & $\operatorname{MIC}\left(\times 10^{-7} \mathrm{~m} \mathrm{~s}^{-1}\right)$ & 7.32 & 8.47 & 8.63 & & \\
\hline & & $P\left(m^{3} m^{-3}\right)-1 s t$ layer & 0.52 & 0.59 & 0.52 & & \\
\hline & & $P\left(m^{3} m^{-3}\right)-2$ nd layer & 0.54 & 0.48 & 0.54 & & \\
\hline & & $P\left(m^{3} m^{-3}\right)$ - 3rd layer & 0.43 & 0.56 & 0.60 & & \\
\hline & \multirow{6}{*}{ ITA5 } & $\mathrm{K}_{\mathrm{Is}}\left(\mathrm{x} 10^{-5} \mathrm{~m} \mathrm{~s}^{-1}\right)$ & 3.66 & 5.41 & 1.87 & \multirow{6}{*}{0.51} & \multirow{6}{*}{10} \\
\hline & & EDR (dimensionless) & 0.43 & 0.24 & 0.43 & & \\
\hline & & $\operatorname{MIC}\left(\times 10^{-7} \mathrm{~m} \mathrm{~s}^{-1}\right)$ & 8.47 & 8.47 & 8.63 & & \\
\hline & & $P\left(m^{3} m^{-3}\right)-1 s t$ layer & 0.57 & 0.54 & 0.52 & & \\
\hline & & $P\left(m^{3} m^{-3}\right)-2$ nd layer & 0.54 & 0.53 & 0.59 & & \\
\hline & & $P\left(m^{3} m^{-3}\right)$ - 3rd layer & 0.38 & 0.56 & 0.55 & & \\
\hline \multirow{30}{*}{ RIV } & \multirow{6}{*}{ RIV1 } & $\mathrm{K}_{\mathrm{Is}}\left(\mathrm{x} 10^{-5} \mathrm{~m} \mathrm{~s}^{-1}\right)$ & 2.76 & 5.11 & 1.57 & \multirow{6}{*}{0.56} & \multirow{6}{*}{14} \\
\hline & & EDR (dimensionless) & 0.43 & 0.14 & 0.43 & & \\
\hline & & $\operatorname{MIC}\left(\times 10^{-7} \mathrm{~m} \mathrm{~s}^{-1}\right)$ & 6.74 & 8.47 & 8.63 & & \\
\hline & & $P\left(m^{3} m^{-3}\right)-1 s t$ layer & 0.52 & 0.59 & 0.52 & & \\
\hline & & $P\left(m^{3} m^{-3}\right)-2$ nd layer & 0.59 & 0.48 & 0.54 & & \\
\hline & & $P\left(m^{3} m^{-3}\right)$ - 3rd layer & 0.48 & 0.56 & 0.55 & & \\
\hline & & $\mathrm{K}_{\mathrm{Is}}\left(\mathrm{x} 10^{-5} \mathrm{~m} \mathrm{~s}^{-1}\right)$ & 3.36 & 5.11 & 2.17 & & \\
\hline & & EDR (dimensionless) & 0.33 & 0.14 & 0.43 & & \\
\hline & RIV? & $\operatorname{MIC}\left(x 10^{-7} \mathrm{~m} \mathrm{~s}^{-1}\right)$ & 7.90 & 8.47 & 8.63 & 045 & 7 \\
\hline & RIV 2 & $P\left(m^{3} m^{-3}\right)-1 s t$ layer & 0.47 & 0.59 & 0.52 & 0.43 & 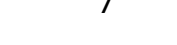 \\
\hline & & $P\left(m^{3} m^{-3}\right)-2$ nd layer & 0.54 & 0.48 & 0.59 & & \\
\hline & & $P\left(m^{3} m^{-3}\right)$ - 3rd layer & 0.48 & 0.56 & 0.60 & & \\
\hline & & $\mathrm{K}_{\mathrm{Is}}\left(\mathrm{x} 10^{-5} \mathrm{~m} \mathrm{~s}^{-1}\right)$ & 3.06 & 5.11 & 2.17 & & \\
\hline & & EDR (dimensionless) & 0.33 & 0.14 & 0.43 & & \\
\hline & DIV/2 & $\operatorname{MIC}\left(x 10^{-7} \mathrm{~m} \mathrm{~s}^{-1}\right)$ & 7.32 & 8.47 & 8.63 & 015 & 7 \\
\hline & Riv3 & $P\left(m^{3} m^{-3}\right)-1 s t$ layer & 0.52 & 0.59 & 0.52 & 0.45 & $y$ \\
\hline & & $P\left(m^{3} m^{-3}\right)-2$ nd layer & 0.54 & 0.48 & 0.59 & & \\
\hline & & $P\left(m^{3} m^{-3}\right)$ - 3rd layer & 0.43 & 0.56 & 0.60 & & \\
\hline & & $\mathrm{K}_{\mathrm{Is}}\left(\mathrm{x} 10^{-5} \mathrm{~m} \mathrm{~s}^{-1}\right)$ & 3.06 & 5.11 & 2.17 & & \\
\hline & & EDR (dimensionless) & 0.33 & 0.14 & 0.43 & & \\
\hline & DIVA & $\operatorname{MIC}\left(\times 10^{-7} \mathrm{~m} \mathrm{~s}^{-1}\right)$ & 7.32 & 8.47 & 8.63 & 015 & 7 \\
\hline & RIV4 & $P\left(m^{3} m^{-3}\right)-1 s t$ layer & 0.52 & 0.59 & 0.52 & 0.45 & 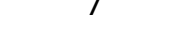 \\
\hline & & $P\left(m^{3} m^{-3}\right)-2$ nd layer & 0.54 & 0.48 & 0.54 & & \\
\hline & & $P\left(m^{3} m^{-3}\right)$ - 3rd layer & 0.43 & 0.56 & 0.60 & & \\
\hline & & $\mathrm{K}_{\mathrm{Is}}\left(\mathrm{x} 10^{-5} \mathrm{~m} \mathrm{~s}^{-1}\right)$ & 3.66 & 5.41 & 1.87 & & \\
\hline & & EDR (dimensionless) & 0.43 & 0.24 & 0.43 & & \\
\hline & DIV/5 & $\operatorname{MIC}\left(\times 10^{-7} \mathrm{~m} \mathrm{~s}^{-1}\right)$ & 8.47 & 8.47 & 8.63 & 015 & 8 \\
\hline & RIVS & $P\left(m^{3} m^{-3}\right)-1$ st layer & 0.57 & 0.54 & 0.52 & 0.45 & 8 \\
\hline & & $P\left(m^{3} m^{-3}\right)-2 n d$ layer & 0.54 & 0.53 & 0.59 & & \\
\hline & & $P\left(m^{3} m^{-3}\right)-3 r d$ layer & 0.38 & 0.56 & 0.55 & & \\
\hline
\end{tabular}


Table 4 - Calibrated parameters and performance indexes for the five clusters of each watershed upstream from the USM station

\begin{tabular}{|c|c|c|c|c|c|c|c|}
\hline Basin & Cluster & Parameter & Ultisol & Oxisol & Interceptisol & NS & PBIAS (\%) \\
\hline & \multirow{6}{*}{ FLA1 } & $\mathrm{K}_{\mathrm{ls}}\left(\mathrm{x} 10^{-5} \mathrm{~m} \mathrm{~s}^{-1}\right)$ & 3.01 & 3.91 & 8.41 & \multirow{6}{*}{0.45} & \multirow{6}{*}{4} \\
\hline & & EDR (dimensionless) & 0.44 & 0.44 & 0.14 & & \\
\hline & & $\operatorname{MIC}\left(x 10^{-7} \mathrm{~m} \mathrm{~s}^{-1}\right)$ & 8.47 & 4.00 & 8.05 & & \\
\hline & & $P\left(m^{3} m^{-3}\right)-1 s t$ layer & 0.34 & 0.49 & 0.59 & & \\
\hline & & $P\left(m^{3} m^{-3}\right)-2 n d$ layer & 0.43 & 0.58 & 0.58 & & \\
\hline & & $P\left(m^{3} m^{-3}\right)-3 r d$ layer & 0.46 & 0.56 & 0.56 & & \\
\hline & \multirow{6}{*}{ FLA2 } & $\mathrm{K}_{\mathrm{Is}}\left(\mathrm{x} 10^{-5} \mathrm{~m} \mathrm{~s}^{-1}\right)$ & 3.01 & 3.91 & 7.81 & \multirow{6}{*}{0.44} & \multirow{6}{*}{3} \\
\hline & & EDR (dimensionless) & 0.44 & 0.44 & 0.14 & & \\
\hline & & $\operatorname{MIC}\left(x 10^{-7} \mathrm{~m} \mathrm{~s}^{-1}\right)$ & 8.47 & 4.00 & 8.05 & & \\
\hline & & $P\left(m^{3} m^{-3}\right)-1$ st layer & 0.34 & 0.49 & 0.54 & & \\
\hline & & $P\left(m^{3} m^{-3}\right)-2 n d$ layer & 0.48 & 0.58 & 0.58 & & \\
\hline \multirow{13}{*}{ FLA } & & $P\left(m^{3} m^{-3}\right)-3 r d$ layer & 0.46 & 0.56 & 0.56 & & \\
\hline & \multirow{6}{*}{ FLA3 } & $\mathrm{K}_{\mathrm{Is}}\left(\mathrm{x} 10^{-5} \mathrm{~m} \mathrm{~s}^{-1}\right)$ & 2.71 & 3.01 & 5.41 & \multirow{6}{*}{0.39} & \multirow{6}{*}{6} \\
\hline & & EDR (dimensionless) & 0.44 & 0.44 & 0.14 & & \\
\hline & & $\operatorname{MIC}\left(x 10^{-7} \mathrm{~m} \mathrm{~s}^{-1}\right)$ & 6.16 & 4.00 & 7.74 & & \\
\hline & & $P\left(m^{3} m^{-3}\right)-1 s t$ layer & 0.49 & 0.54 & 0.59 & & \\
\hline & & $P\left(m^{3} m^{-3}\right)-2 n d$ layer & 0.53 & 0.58 & 0.58 & & \\
\hline & & $P\left(m^{3} m^{-3}\right)-3 r d$ layer & 0.51 & 0.56 & 0.56 & & \\
\hline & \multirow{6}{*}{ FLA4 } & $\mathrm{K}_{\mathrm{ls}}\left(\mathrm{x} 10^{-5} \mathrm{~m} \mathrm{~s}^{-1}\right)$ & 3.01 & 3.91 & 7.51 & \multirow{6}{*}{0.43} & \multirow{6}{*}{0} \\
\hline & & EDR (dimensionless) & 0.44 & 0.44 & 0.14 & & \\
\hline & & $\operatorname{MIC}\left(x 10^{-7} \mathrm{~m} \mathrm{~s}^{-1}\right)$ & 8.47 & 4.00 & 7.47 & & \\
\hline & & $P\left(m^{3} m^{-3}\right)-1 s t$ layer & 0.34 & 0.49 & 0.54 & & \\
\hline & & $P\left(m^{3} m^{-3}\right)-2 n d$ layer & 0.48 & 0.58 & 0.58 & & \\
\hline & & $P\left(m^{3} m^{-3}\right)-3 r d$ layer & 0.51 & 0.56 & 0.56 & & \\
\hline
\end{tabular}

Continuation... 


\begin{tabular}{|c|c|c|c|c|c|c|c|}
\hline Basin & Cluster & Parameter & Ultisol & Oxisol & Interceptisol & NS & PBIAS (\%) \\
\hline & \multirow{6}{*}{ FLA5 } & $\mathrm{K}_{\mathrm{Is}}\left(\mathrm{x} 10^{-5} \mathrm{~m} \mathrm{~s}^{-1}\right)$ & 3.01 & 3.31 & 5.71 & \multirow{6}{*}{0.46} & \multirow{6}{*}{8} \\
\hline & & EDR (dimensionless) & 0.44 & 0.44 & 0.14 & & \\
\hline & & $\operatorname{MIC}\left(\times 10^{-7} \mathrm{~m} \mathrm{~s}^{-1}\right)$ & 6.74 & 4.00 & 8.05 & & \\
\hline & & $P\left(m^{3} m^{-3}\right)-1 s t$ layer & 0.44 & 0.54 & 0.59 & & \\
\hline & & $P\left(m^{3} m^{-3}\right)-2$ nd layer & 0.53 & 0.53 & 0.53 & & \\
\hline & & $P\left(m^{3} m^{-3}\right)$ - 3rd layer & 0.46 & 0.56 & 0.46 & & \\
\hline \multirow{18}{*}{ CAS } & \multirow{6}{*}{ CAS1 } & $\mathrm{K}_{\mathrm{Is}}\left(\mathrm{x} 10^{-5} \mathrm{~m} \mathrm{~s}^{-1}\right)$ & 4.81 & 6.01 & 2.11 & \multirow{6}{*}{0.43} & \multirow{6}{*}{11} \\
\hline & & EDR (dimensionless) & 0.24 & 0.14 & 0.44 & & \\
\hline & & $\operatorname{MIC}\left(\times 10^{-7} \mathrm{~m} \mathrm{~s}^{-1}\right)$ & 4.42 & 3.42 & 4.58 & & \\
\hline & & $P\left(m^{3} m^{-3}\right)-1 s t$ layer & 0.59 & 0.54 & 0.59 & & \\
\hline & & $P\left(m^{3} m^{-3}\right)-2$ nd layer & 0.58 & 0.58 & 0.53 & & \\
\hline & & $P\left(m^{3} m^{-3}\right)-3 r d$ layer & 0.41 & 0.46 & 0.43 & & \\
\hline & \multirow{6}{*}{ CAS2 } & $\mathrm{K}_{\mathrm{Is}}\left(\mathrm{x} 10^{-5} \mathrm{~m} \mathrm{~s}^{-1}\right)$ & 4.81 & 6.01 & 2.11 & \multirow{6}{*}{0.41} & \multirow{6}{*}{8} \\
\hline & & EDR (dimensionless) & 0.24 & 0.34 & 0.44 & & \\
\hline & & $\operatorname{MIC}\left(\times 10^{-7} \mathrm{~m} \mathrm{~s}^{-1}\right)$ & 4.42 & 3.42 & 4.58 & & \\
\hline & & $P\left(m^{3} m^{-3}\right)-1 s t$ layer & 0.59 & 0.59 & 0.49 & & \\
\hline & & $P\left(m^{3} m^{-3}\right)-2$ nd layer & 0.58 & 0.53 & 0.53 & & \\
\hline & & $P\left(m^{3} m^{-3}\right)$ - 3rd layer & 0.41 & 0.56 & 0.56 & & \\
\hline & \multirow{6}{*}{ CAS3 } & $\mathrm{K}_{\mathrm{Is}}\left(\mathrm{x} 10^{-5} \mathrm{~m} \mathrm{~s}^{-1}\right)$ & 4.21 & 4.51 & 2.11 & \multirow{6}{*}{0.37} & \multirow{6}{*}{12} \\
\hline & & EDR (dimensionless) & 0.24 & 0.14 & 0.24 & & \\
\hline & & MIC (x10-7 m s-1) & 3.84 & 3.42 & 3.42 & & \\
\hline & & $P(m 3$ m-3) - 1st layer & 0.59 & 0.59 & 0.59 & & \\
\hline & & P (m3 m-3) - 2nd layer & 0.48 & 0.53 & 0.58 & & \\
\hline & & P (m3 m-3) - 3rd layer & 0.46 & 0.56 & 0.51 & & \\
\hline
\end{tabular}

Continuation... 
Table 4 - Continuation...

\begin{tabular}{|c|c|c|c|c|c|c|c|}
\hline Basin & Cluster & Parameter & Ultisol & Oxisol & Interceptisol & NS & PBIAS (\%) \\
\hline & \multirow{6}{*}{ CAS4 } & Kls (x10-5 m s-1) & 481 & 6.01 & 2.11 & \multirow{6}{*}{0.43} & \multirow{6}{*}{11} \\
\hline & & EDR (dimensionless) & 0.24 & 0.24 & 0.44 & & \\
\hline & & MIC (x10-7 m s-1) & 4.42 & 3.42 & 4.58 & & \\
\hline & & $P(m 3 m-3)-1$ st layer & 0.59 & 0.59 & 0.59 & & \\
\hline & & P (m3 m-3) - 2nd layer & 0.53 & 0.58 & 0.53 & & \\
\hline & & P (m3 m-3) - 3rd layer & 0.41 & 0.46 & 0.46 & & \\
\hline & \multirow{6}{*}{ CAS5 } & Kls (x10-5 m s-1) & 4.21 & 4.81 & 1.81 & \multirow{6}{*}{0.38} & \multirow{6}{*}{11} \\
\hline & & EDR (dimensionless) & 0.24 & 0.14 & 0.24 & & \\
\hline & & MIC (x10-7 m s-1) & 3.84 & 3.42 & 3.42 & & \\
\hline & & $P(m 3$ m-3) - 1st layer & 0.59 & 0.59 & 0.59 & & \\
\hline & & P (m3 m-3) - 2nd layer & 0.48 & 0.53 & 0.58 & & \\
\hline & & $P(m 3$ m-3) - 3rd layer & 0.46 & 0.56 & 0.51 & & \\
\hline \multirow{12}{*}{ USM } & \multirow{6}{*}{ USM1 } & Kls (x10-5 m s-1) & 2.76 & 3.66 & 3.37 & \multirow{6}{*}{0.40} & \multirow{6}{*}{29} \\
\hline & & EDR (dimensionless) & 0.33 & 0.43 & 0.43 & & \\
\hline & & MIC (x10-7 m s-1) & 8.47 & 8.63 & 4.00 & & \\
\hline & & $P(m 3$ m-3) - 1st layer & 0.57 & 0.37 & 0.52 & & \\
\hline & & $P(m 3$ m-3) - 2nd layer & 0.54 & 0.39 & 0.49 & & \\
\hline & & P (m3 m-3) - 3rd layer & 0.58 & 0.48 & 0.50 & & \\
\hline & \multirow{6}{*}{ USM2 } & Kls (x10-5 m s-1) & 2.76 & 3.66 & 3.67 & \multirow{6}{*}{0.42} & \multirow{6}{*}{26} \\
\hline & & EDR (dimensionless) & 0.43 & 0.33 & 0.43 & & \\
\hline & & MIC (x10-7 m s-1) & 8.47 & 8.63 & 4.00 & & \\
\hline & & $P(m 3$ m-3) - 1st layer & 0.57 & 0.37 & 0.52 & & \\
\hline & & P (m3 m-3) - 2nd layer & 0.54 & 0.39 & 0.49 & & \\
\hline & & $P(m 3$ m-3) - 3rd layer & 0.58 & 0.48 & 0.50 & & \\
\hline
\end{tabular}

Continuation... 


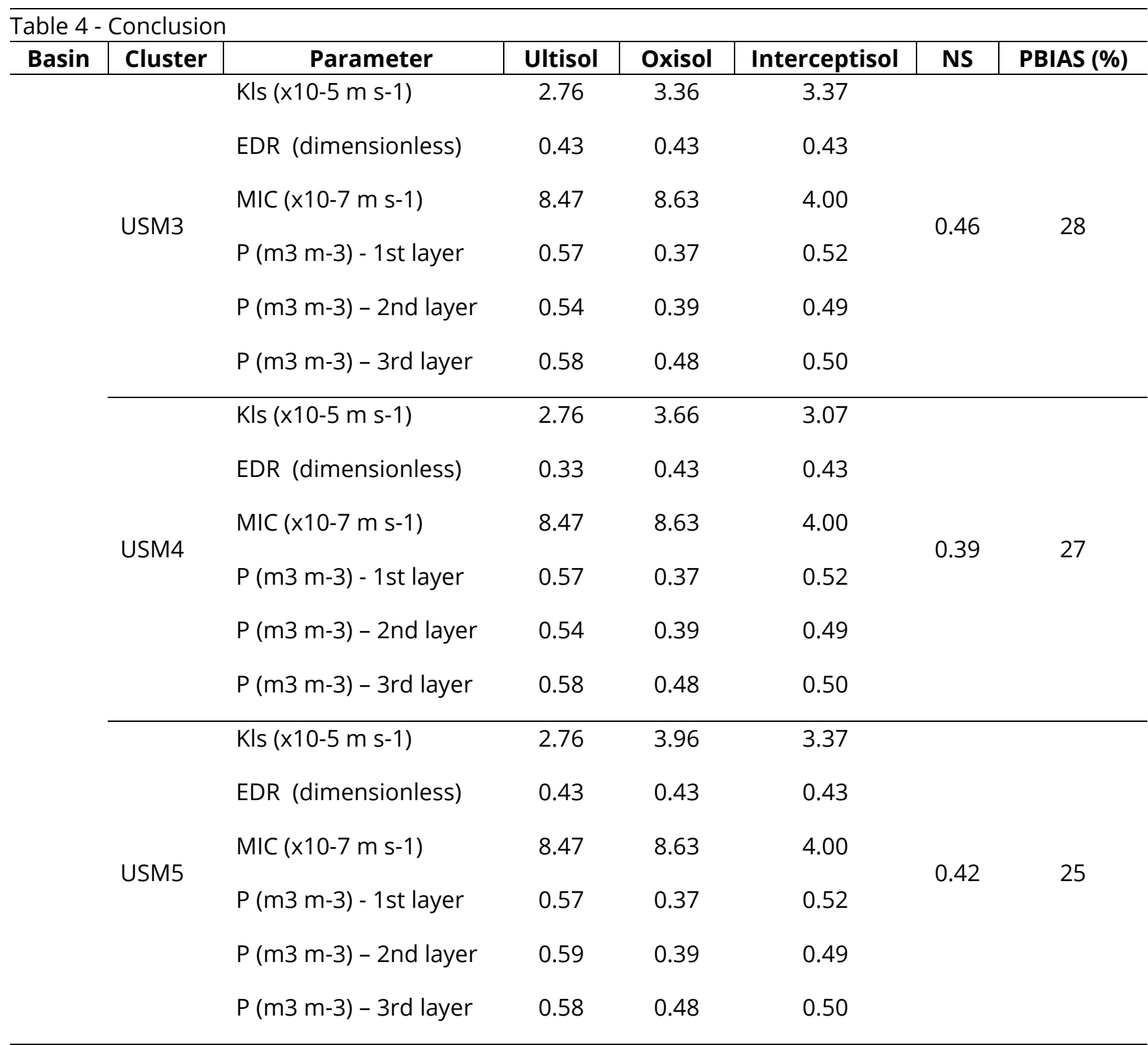

Table 5 - Calibrated parameters and performance indexes for the five clusters of UPA

\begin{tabular}{|c|c|c|c|c|c|c|}
\hline Cluster & Parameter & Ultisol & Oxisol & Interceptisol & NS & PBIAS (\%) \\
\hline \multirow{6}{*}{ UPA1 } & $\mathrm{K}_{\mathrm{Is}}\left(\mathrm{x} 10^{-5} \mathrm{~m} \mathrm{~s}^{-1}\right)$ & 3.06 & 3.31 & 2.17 & \multirow{6}{*}{0.57} & \multirow{6}{*}{26} \\
\hline & EDR (dimensionless) & 0.13 & 0.14 & 0.33 & & \\
\hline & $\operatorname{MIC}\left(x 10^{-7} \mathrm{~m} \mathrm{~s}^{-1}\right)$ & 5.00 & 5.58 & 8.58 & & \\
\hline & $P\left(m^{3} m^{-3}\right)-1 s t$ layer & 0.52 & 0.54 & 0.52 & & \\
\hline & $P\left(m^{3} m^{-3}\right)-2 n d$ layer & 0.54 & 0.53 & 0.54 & & \\
\hline & $P\left(m^{3} m^{-3}\right)$ - 3rd layer & 0.48 & 0.56 & 0.55 & & \\
\hline
\end{tabular}




\begin{tabular}{|c|c|c|c|c|c|c|}
\hline Cluster & Parameter & Ultisol & Oxisol & Interceptisol & NS & PBIAS (\%) \\
\hline \multirow{6}{*}{ UPA2 } & $\mathrm{K}_{\mathrm{Is}}\left(\mathrm{x} 10^{-5} \mathrm{~m} \mathrm{~s}^{-1}\right)$ & 2.46 & 2.71 & 2.17 & \multirow{6}{*}{0.58} & \multirow{6}{*}{22} \\
\hline & EDR (dimensionless) & 0.23 & 0.14 & 0.23 & & \\
\hline & $\operatorname{MIC}\left(x 10^{-7} \mathrm{~m} \mathrm{~s}^{-1}\right)$ & 5.00 & 4.42 & 4.58 & & \\
\hline & $P\left(m^{3} m^{-3}\right)-1 s t$ layer & 0.52 & 0.54 & 0.52 & & \\
\hline & $P\left(m^{3} m^{-3}\right)-2 n d$ layer & 0.49 & 0.53 & 0.54 & & \\
\hline & $P\left(m^{3} m^{-3}\right)-3 r d$ layer & 0.48 & 0.51 & 0.55 & & \\
\hline \multirow{6}{*}{ UPA3 } & $\mathrm{K}_{\mathrm{ls}}\left(\mathrm{x} 10^{-5} \mathrm{~m} \mathrm{~s}^{-1}\right)$ & 2.76 & 2.71 & 2.17 & \multirow{6}{*}{0.58} & \multirow{6}{*}{23} \\
\hline & EDR (dimensionless) & 0.23 & 0.14 & 0.23 & & \\
\hline & $\operatorname{MIC}\left(x 10^{-7} \mathrm{~m} \mathrm{~s}^{-1}\right)$ & 5.00 & 4.42 & 4.58 & & \\
\hline & $P\left(m^{3} m^{-3}\right)-1 s t$ layer & 0.52 & 0.54 & 0.52 & & \\
\hline & $P\left(m^{3} m^{-3}\right)-2 n d$ layer & 0.49 & 0.53 & 0.54 & & \\
\hline & $P\left(m^{3} m^{-3}\right)-3 r d$ layer & 0.48 & 0.51 & 0.55 & & \\
\hline \multirow{6}{*}{ UPA4 } & $\mathrm{K}_{\mathrm{ls}}\left(\mathrm{x} 10^{-5} \mathrm{~m} \mathrm{~s}^{-1}\right)$ & 2.76 & 3.01 & 2.17 & \multirow{6}{*}{0.59} & \multirow{6}{*}{27} \\
\hline & EDR (dimensionless) & 0.23 & 0.14 & 0.23 & & \\
\hline & $\operatorname{MIC}\left(x 10^{-7} \mathrm{~m} \mathrm{~s}^{-1}\right)$ & 5.00 & 4.42 & 4.58 & & \\
\hline & $P\left(m^{3} m^{-3}\right)-1$ st layer & 0.52 & 0.54 & 0.52 & & \\
\hline & $P\left(m^{3} m^{-3}\right)-2 n d$ layer & 0.54 & 0.53 & 0.54 & & \\
\hline & $P\left(m^{3} m^{-3}\right)-3 r d$ layer & 0.43 & 0.51 & 0.50 & & \\
\hline \multirow{6}{*}{ UPA5 } & $\mathrm{K}_{\mathrm{Is}}\left(\mathrm{x} 10^{-5} \mathrm{~m} \mathrm{~s}^{-1}\right)$ & 2.76 & 3.01 & 2.17 & \multirow{6}{*}{0.61} & \multirow{6}{*}{25} \\
\hline & EDR (dimensionless) & 0.23 & 0.24 & 0.33 & & \\
\hline & $\operatorname{MIC}\left(x 10^{-7} \mathrm{~m} \mathrm{~s}^{-1}\right)$ & 5.00 & 4.42 & 4.58 & & \\
\hline & $P\left(m^{3} m^{-3}\right)-1 s t$ layer & 0.52 & 0.54 & 0.52 & & \\
\hline & $P\left(m^{3} m^{-3}\right)-2 n d$ layer & 0.54 & 0.53 & 0.54 & & \\
\hline & $P\left(m^{3} m^{-3}\right)$ - 3rd layer & 0.43 & 0.56 & 0.50 & & \\
\hline
\end{tabular}

\subsection{Transfer of calibrated parameters}

Figures 4 to 6 present the evaluation of the cluster transfer (only NS index). The black bars represent the application of clusters in the same watershed where they were calibrated, only being presented for comparison. The red bars represent clusters whose efficiency is unsatisfactory for the watershed under analysis. Blue bars refer to clusters with satisfactory performance (NS $\geq 0.3$ ). 
Figure 4 - Efficiency of calibration transfer to the watersheds upstream from the RIV

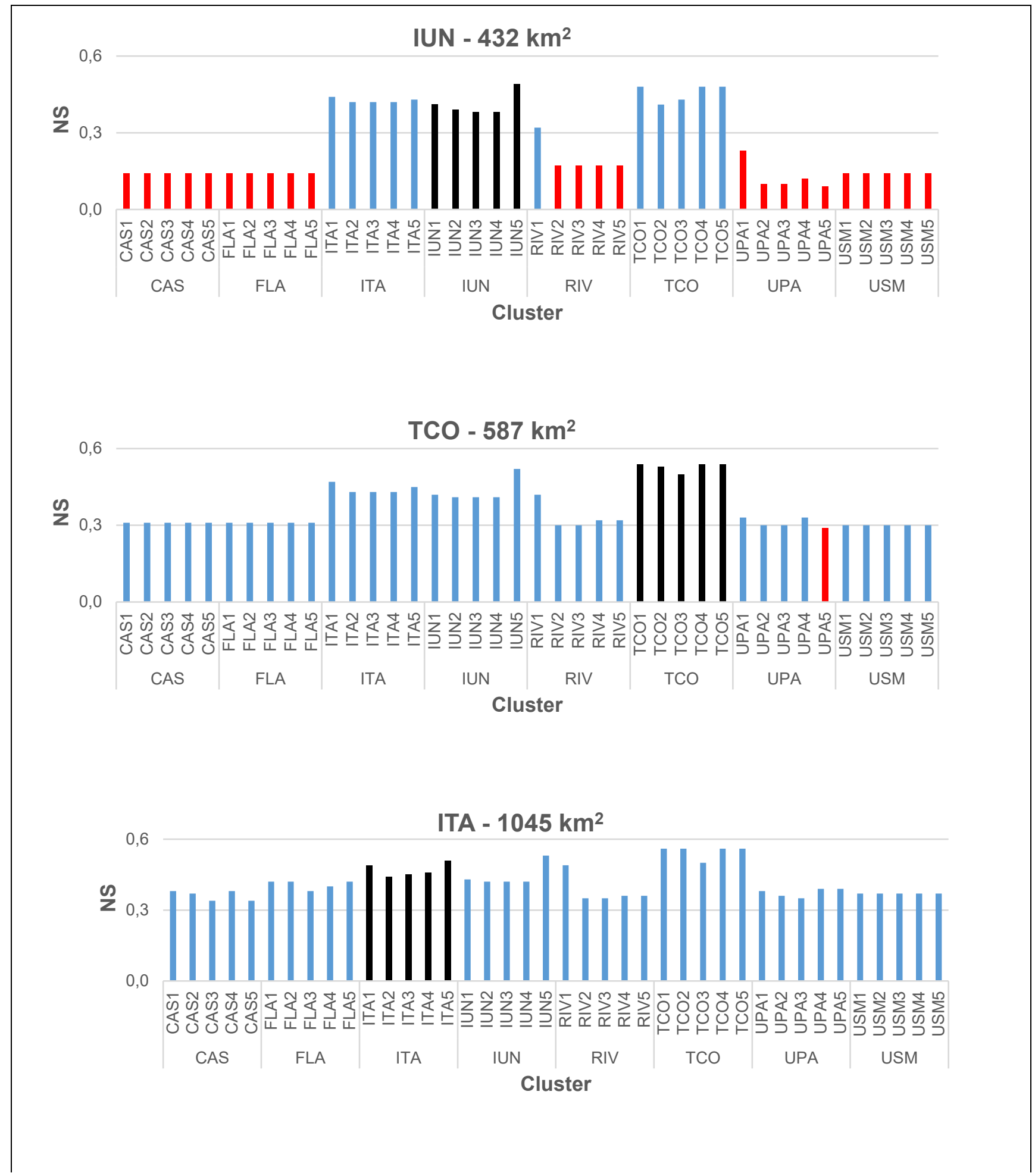




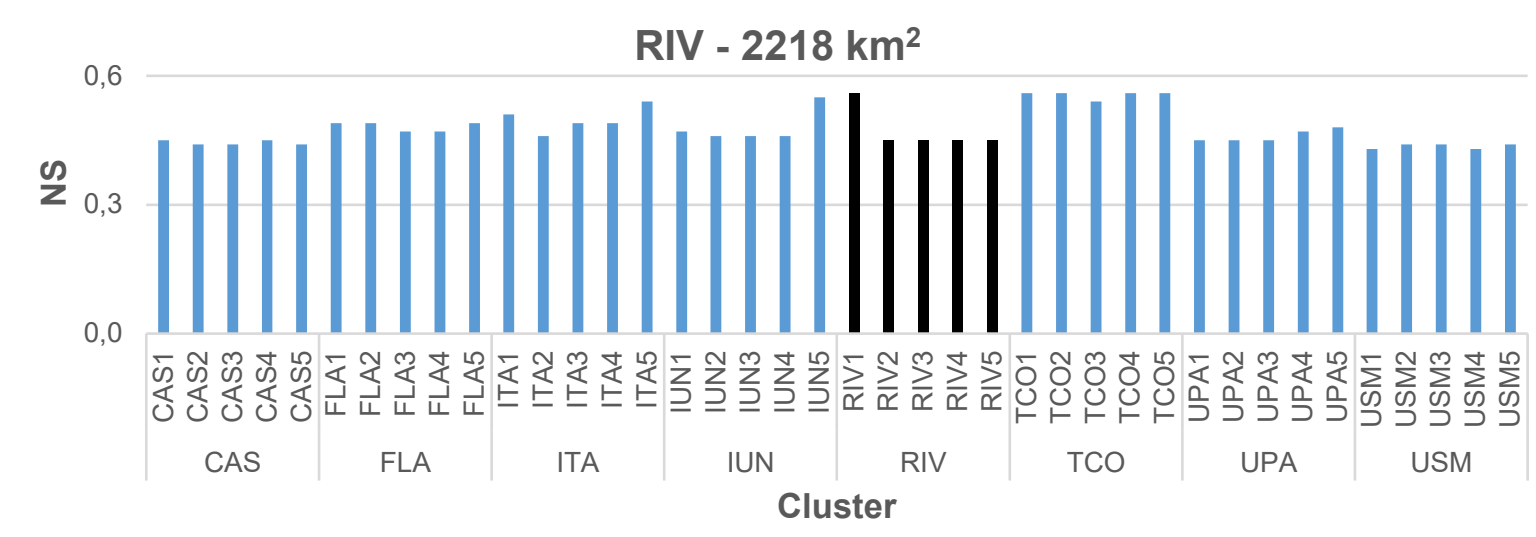

Figure 5 - Efficiency of calibration transfer to the watersheds upstream from USM

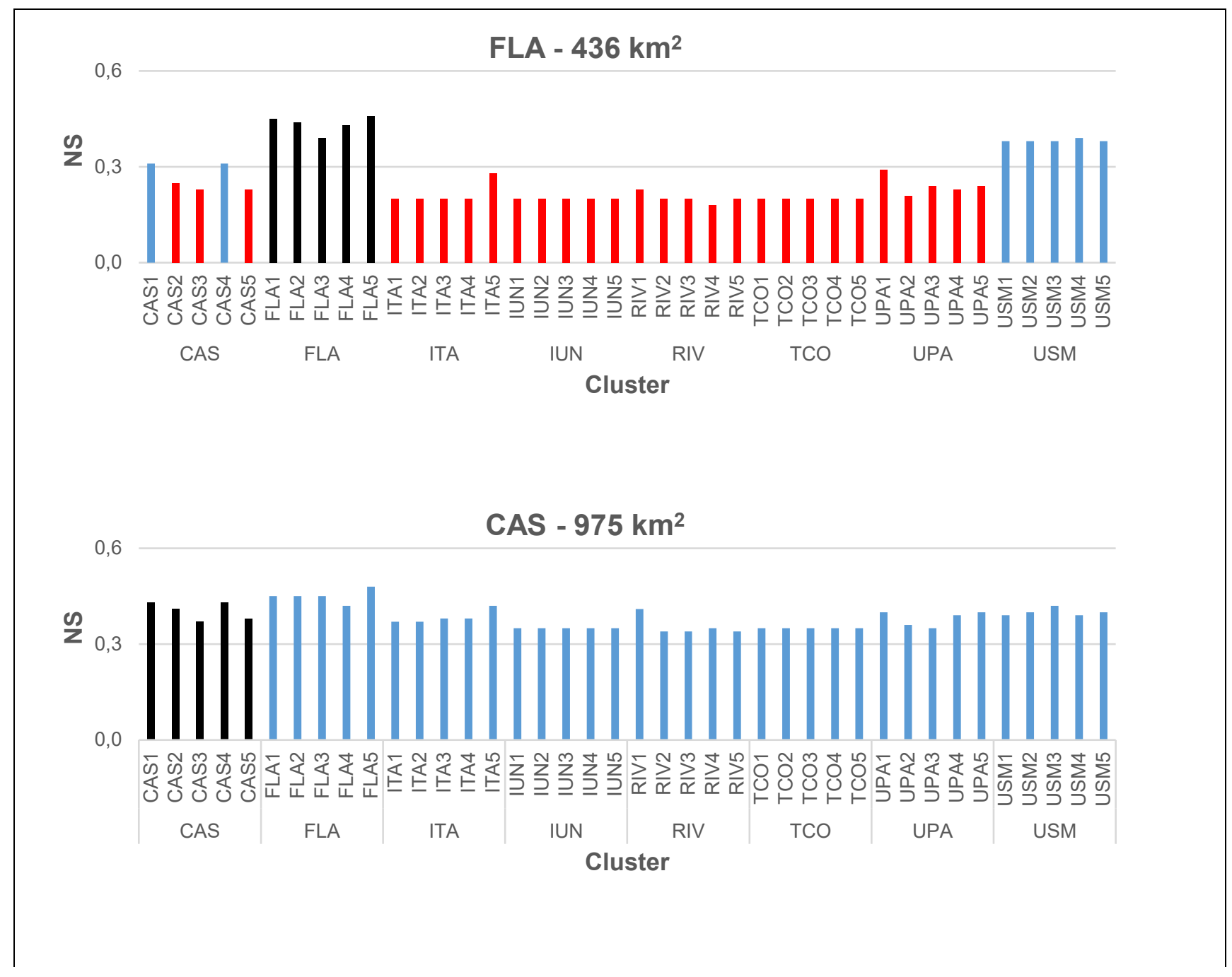




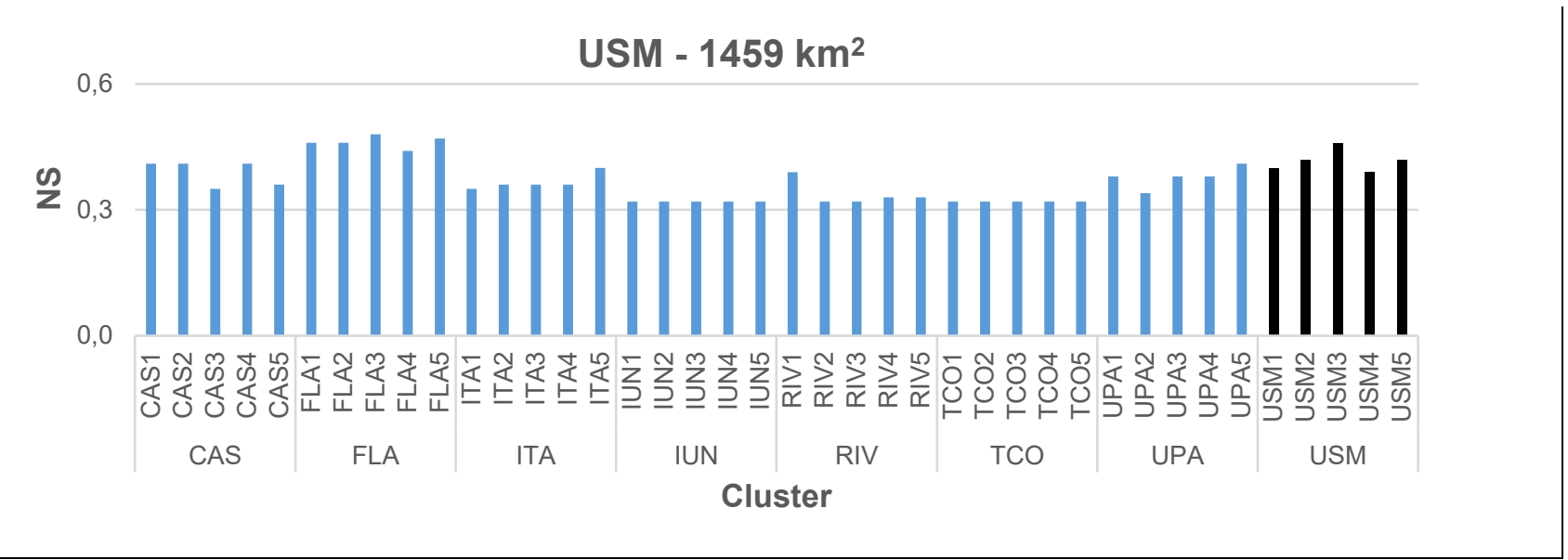

Figure 6 - Efficiency of calibration transfer for the UPA watershed

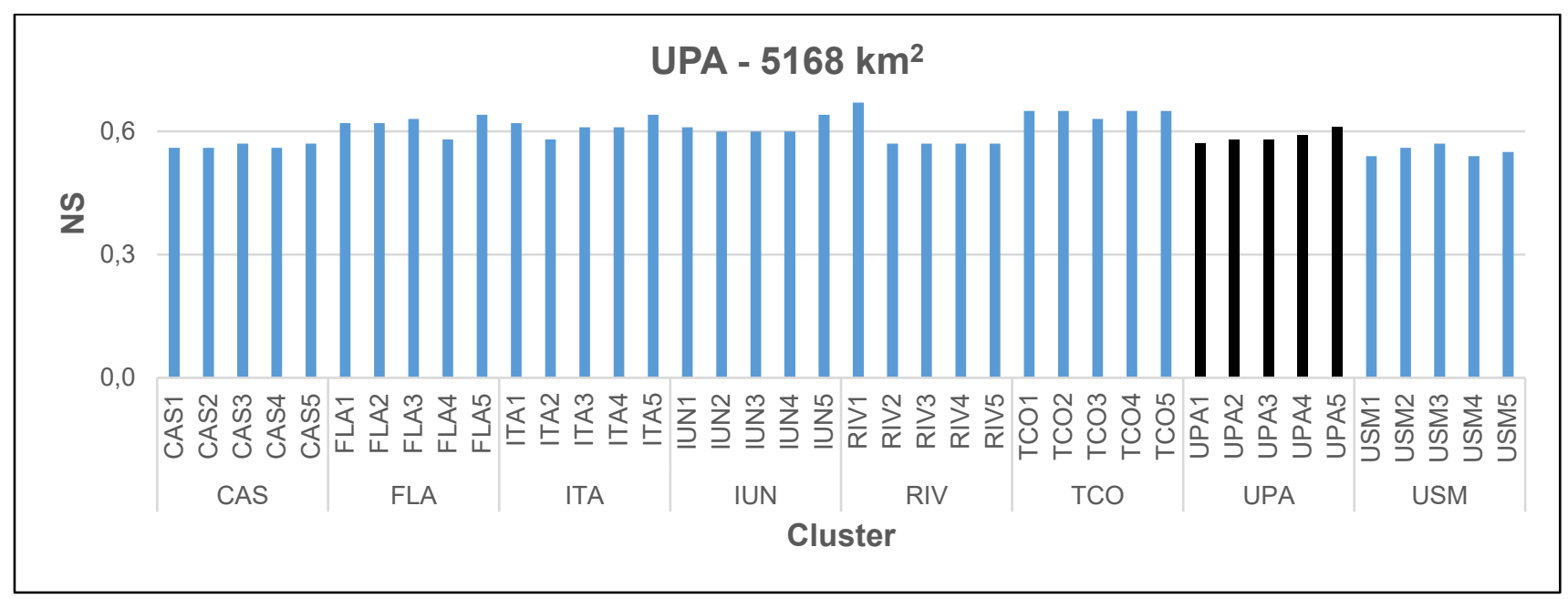

\section{DISCUSSION}

\subsection{Calibration of each watershed}

The calibrated parameters (Table 3 to Table 5) were within the magnitude of its initial values (Table 2). Average $\mathrm{K}_{\mathrm{ls}}\left(5.14 \cdot 10^{-5} \mathrm{~m} \mathrm{~s}^{-1}\right)$ and $\mathrm{P}\left(0.48 \mathrm{~m}^{3} \mathrm{~m}^{-3}\right)$ were close to those calibrated for the Mae Chaem Basin in Thailand (THANAPAKPAWIN et al., 2007). Average EDR (0.33) was similar to watersheds in the Amazon Basin (CUARTAS et al., 2012). Only MIC was considerably lower than values presented in 
other DHSVM applications in which its magnitude was of some thousands of $\mathrm{mm}$ day $^{-1}$ (ALVARENGA et al., 2016, 2017; CUO et al., 2006). The MIC magnitude in this paper was a few tens of $\mathrm{mm} \mathrm{day}^{-1}$, therefore more consistent with reality. The maximum soil depth in the cited papers was set to less than $7.2 \mathrm{~m}$, compensating the higher MIC values to simulate the streamflow due to the smaller soil depth leading to a decrease in the maximum soil storage, and consequently to greater runoff and streamflow. It is generally observed that the calibrated parameters of the clusters were close for each watershed, and did not present a trend when comparing the five "best" solutions for each soil class. The parameters with the most significant variation among clusters were MIC and P. When the MIC had its value increased the $P$ was reduced, and vice versa. This behavior is explained by the fact that both influence the water storage in the soil in different ways, and consequently in the runoff and baseflow.

Regarding the modeling efficiency, the NS values of the clusters of each watershed only presented small differences. These differences ranged from 0.37 to 0.43 at CAS, with the worse performance (Table 4); and from 0.57 to 0.61 at UPA, with the best performance (Table 5). The existence of such small differences in NS confirms the non-linear characteristics of the calibration problem in the study area, thus indicating that the calibration problem has more than one feasible solution. This fact corroborates the previously highlighted results in other works with hydrological models (DUAN; SOROOSHIAN; GUPTA, 1992). Moreover, the proposed methodology of adjusting more than one set of calibrated parameters (cluster) may have the potential to transfer one or more of these clusters to similar catchments downstream or upstream of the calibrated watersheds, as demonstrated later in this paper.

The calibrated parameters in some of the clusters of each watershed are quite close and result in virtually identical NS, as in the example of the RIV2, RIV3, RIV4, and RIV5 clusters (Table 3). This fact has its origin in the calibration method itself; the calibration system only explored the search space parameter values in 
the vicinity of a good location, for which NS is acceptable, although there is a better performing cluster (RIV1). In other situations, as in UPA (Table 5), the optimal solution is close to those obtained for the clusters and in the same region of the search space. In both cases, the number of random solutions generated and verified in the process was not ideal due to the computational cost of the DHSVM model. The CS metaheuristic has likely improperly explored other promising regions of the search space, thus not allowing the calibration of even better solutions. However, the differences among calibrated parameters for the majority of the clusters (ITA on Table 3, for example) shows that several "optimal" solutions were found, making all the parameter sets feasible.

The watersheds of Group I (upstream of RIV) presented better calibration, with NS varying between 0.38 and 0.56 in relation to Group II (upstream of USM), in which NS varied between 0.37 and 0.46 . However, the better calibration indexes occurred for RIV (Group III). In this way, we can see a trend of better NS for larger watersheds, such as RIV (NS $=0.56$ ) and UPA (NS $=0.61$ ). IUN (the smallest watershed) presented the worst NS (0.49). In the smaller basins, the hydrological responses are faster, implying greater dynamics for the streamflow, and implicating in a worse performance of hydrologic models (MELO NETO et al., 2014; QI; GRUNWALD, 2005). In larger basins there is a tendency to amortize hydrological response, reflecting in better calibration, as found in other Brazilian basins (EDUARDO et al., 2016; FUKUNAGA, 2012; MELO NETO et al., 2014) and worldwide (PINIEWSKI; OKRUSZKO, 2011). These results may partly be a consequence of the flow modeling. In DHSVM, the runoff and the baseflow only displace one pixel per time step. This has a direct influence on the displacement time of the water along the watershed. In this case, since the pixel size was the same for all watersheds, the water displacement time in smaller sub-basins was modeled less accurately, implying in a worse estimate of the streamflow in its mouth. Moreover, such behavior may also have part of the explanation in the fact that meteorological data only came from measurements of two stations. So, the wide evapotranspiration 
distribution could not be satisfactory, which may have negatively influenced streamflow modeling for the smaller watersheds.

\subsection{Transfer of calibrated parameters}

The analysis of Figure 4, Figure 5, and Figure 6 allows a series of inferences regarding the feasibility of transferring calibrated parameters among watersheds. First, approximately $81 \%$ of the 280 cluster transfers resulted in NS values above 0.3, denoting satisfactory performance (NOORI; KALIN, 2016).

In $54 \%$ of the cases, the transfer performance was equal to or greater than that of the specific watershed calibration. Similar transfer results were also found in the hydrologic modeling of three watersheds neighboring IRB using the Variable Infiltration Capacity - VIC (CAMPANHARO, 2013). The transferred clusters improved the modeling efficiency in $10 \%$ of the cases (increased NS values), which only happened for CAS (0.43 to 0.48$)$, ITA (0.51 to 0.56$)$, USM (0.46 to 0.48), and UPA (0.61 to 0.67$)$.

In a similar way to (YEN et al., 2015b), the results of this paper demonstrate the viability of transferring calibrated parameters for DHSVM among watersheds of the same basin. As also suggested by (YEN et al., 2015b), it is important to point out that model parameters may not be transferable by using different sources of land use data for the same watershed. However, there are other constraints for the influence of the watershed's drainage area. The 53 transfers whose NS were unsatisfactory (19\% of the total) occurred for watersheds smaller than $600 \mathrm{~km} 2$ (IUN, FLA, and TCO). Of these, 52 were for IUN and FLA, and only one for the largest of the three (TCO). The transfer of clusters to all watersheds with an area greater than $900 \mathrm{~km} 2$ always resulted in satisfactory performance (NS $\geq 0.3$ ).

Moreover, verifying the transfer performance of clusters within Groups I and II, two exciting facts are observed: a) there is a reduction in the NS of the transfer with the decrease in the watershed area; b) the best transferred clusters came from 
a watershed of the same Group; and c) the clusters of the watersheds belonging to a specific Group led to better NS when transferred to other watersheds of the same Group.

For the smaller watersheds (IUN and FLA), the clusters transferred from downstream watersheds did not improve modeling compared to the better performance of the five original calibrations. The same trend was found in the calibration of the SWAT model (EDUARDO et al., 2016), meaning that application of the calibration parameters of larger basins tend to present lower efficiency in small basins. However, the performance of some transferred clusters in the present paper was satisfactory and better than that found by (EDUARDO et al., 2016). Such clusters came from the watersheds immediately downstream of IUN and FLA.

In this way, there exists a relationship between the size of the watershed and the "relative position" of the watershed that provided the best transferred cluster. The streamflows of the smaller watersheds (less than $500 \mathrm{~km} 2$ ), namely IUN and FLA, were better modeled by clusters from watersheds immediately downstream. Intermediate area watersheds (between 500 and $1400 \mathrm{~km} 2$ ) had their flows best simulated by the clusters of watersheds immediately upstream. Finally, the best cluster in the larger basins (greater than $1400 \mathrm{~km} 2$ ) came from a basin upstream. These results indicate the same tendency evidenced by Eduardo et al. (2016), being that calibrated data for upstream sub-basins are feasible for downstream subbasins, with better performances for larger basins.

\section{CONCLUSIONS}

An exciting innovation of the proposed method is the adjustment of several sets of calibrated parameters (clusters) for each watershed. Other methods (BRIGHENTI; BONUMÁ; CHAFFE, 2016; PINIEWSKI; OKRUSZKO, 2011) only consider a single "optimal" set of calibrated parameters for each watershed. 
The results obtained in the present paper show that the proposed method can be useful in the calibration as well as in extrapolating the parameters of the DHSVM model to regions that do not have information about the parameters of the model. Moreover, it still can improve the calibration result for a given watershed with the application of the calibrated parameters to another catchment.

\section{ACKNOWLEDGMENT}

This study was partially financed by the Conselho Nacional de Desenvolvimento Científico e Tecnológico - Brasil (CNPq) - Finance Code 304916/2017-0.

\section{REFERENCES}

ALVARENGA, L. A. et al. Hydrological responses to climate changes in a headwater watershed. Ciência e Agrotecnologia, v. 40, n. 6, p. 647-657, 2016.

ALVARENGA, L. A. et al. Hydrologic impacts due to the changes in riparian buffer in a headwater watershed. CERNE, v. 23, n. 1, p. 95-102, 2017.

ALVARES, C. A. et al. Köppen's climate classification map for Brazil. Meteorologische Zeitschrift, v. 22, n. 6, p. 711-728, 2013.

ARNOLD, J. G. et al. Hydrological Processes and Model Representation: Impact of Soft Data on Calibration. Transactions of the ASABE, v. 58, n. 6, p. 1637-1660, 2015.

ARSENAULT, R. et al. Comparison of Stochastic Optimization Algorithms in Hydrological Model Calibration. Journal of Hydrologic Engineering, v. 19, n. 7, p. 1374-1384, 2014.

BAI, J.; SHEN, Z.; YAN, T. A comparison of single- and multi-site calibration and validation: a case study of SWAT in the Miyun Reservoir watershed, China. Frontiers of Earth Science, v. 11, n. 3, p. 592-600, 2017.

BECKERS, J.; SMERDON, B.; WILSON, M. Review of hydrologic models for forest management and climate change applications in British Columbia and Alberta. 1. ed. Kamloops: FORREX, 2009. 
BRIGHENTI, T.; BONUMÁ, N. B.; CHAFFE, P. Calibração hierárquica do modelo SWAT em uma bacia hidrográfica Catarinense. Revista Brasileira de Recursos Hídricos, v. 21, n. 1, p. 53-64, 25 fev. 2016.

CAMPANHARO, W. A. Análise do modelo de capacidade de infiltração variável (VIC) para as bacia dos rios Santa Maria da Vitória e Jucu. [s.l.] Universidade Federal do Espírito Santo, 2013.

CUARTAS, L. A. et al. Distributed hydrological modeling of a micro-scale rainforest watershed in Amazonia: Model evaluation and advances in calibration using the new HAND terrain model. Journal of Hydrology, v. 462-463, n. 10, p. 15-27, 2012.

CUO, L. et al. Use of the distributed hydrology soil vegetation model to study road effects on hydrological processes in Pang Khum Experimental Watershed, northern Thailand. Forest Ecology and Management, v. 224, n. 1-5, p. 81-94, 2006.

CUO, L.; GIAMBELLUCA, T. W.; ZIEGLER, A. D. Lumped parameter sensitivity analysis of a distributed hydrological model within tropical and temperate catchments. Hydrological Processes, v. 25, n. 15, p. 2405-2421, 2011.

DAGGUPATI, P. et al. A Recommended Calibration and Validation Strategy for Hydrologic and Water Quality Models. Transactions of the ASABE, v. 58, n. 6, p. 1705-1719, 2015.

DAS, T. et al. Comparison of conceptual model performance using different representations of spatial variability. Journal of Hydrology, v. 356, n. 1-2, p. 106-118, 2008.

DATTA, B. et al. Linking a Simulated Annealing Based Optimization Model with PHT3D Simulation Model for Chemically Reactive Transport Processes to Optimally Characterize Unknown Contaminant Sources in a Former Mine Site in Australia. Journal of Water Resource and Protection, v. 09, n. 05, p. 432-454, 2017.

DUAN, Q.; SOROOSHIAN, S.; GUPTA, H. V. Effective and efficient global optimization for conceptual rainfall-runoff models. Water Resources Research, v. 28, n. 4, p. 1015-1031, abr. 1992.

EASTON, Z. M. et al. Hydrologic assessment of an urban variable source watershed in the northeast United States. Water Resources Research, v. 43, n. 3, p. W03413, 2007.

EDUARDO, E. N. et al. Hydrological simulation as subside for management of surface water resources at the Mortes River Basin. Ciência e Agrotecnologia, v. 40, n. 4, p. 390-404, 2016.

EMBRAPA - EMPRESA BRASILEIRA DE PESQUISA AGROPECUÁRIA. Sistema brasileiro de classificação de solos. 3. ed. Brasília: EMBRAPA - SPI, 2013.

FARJAD, B. et al. An Integrated Modelling System to Predict Hydrological Processes under Climate and Land-Use/Cover Change Scenarios. Water, v. 9, n. 10, p. 767, 2017. 
FUKUNAGA, D. C. Estimação de vazão em bacias hidrográficas do sul do Espírito Santo usando o SWAT. [s.I.] Universidade Federal do Espírito Santo, 2012.

GEOBASES. Sistema integrado de bases geoespaciais do Estado do Espírito Santo. Disponível em: http://www.geobases.es.gov.br/. Acesso em: 7 jul. 2012.

GUPTA, H. V.; SOROOSHIAN, S.; YAPO, P. O. Status of Automatic Calibration for Hydrologic Models: Comparison with Multilevel Expert Calibration. Journal of Hydrologic Engineering, $\mathrm{V}$. 4, n. 2, p. 135-143, 1999.

HASSABALLAH, K. et al. Analysis of streamflow response to land use and land cover changes using satellite data and hydrological modelling: case study of Dinder and Rahad tributaries of the Blue Nile (Ethiopia-Sudan). Hydrology and Earth System Sciences, v. 21, n. 10, p. 52175242, 2017.

INSTITUTO BRASILEIRO DE GEOGRAFIA E ESTATÍSTICA. Manual técnico da vegetação brasileira. 2. ed. Rio de Janeiro: INSTITUTO BRASILEIRO DE GEOGRAFIA E ESTATÍSTICA, 2012.

KAN, G. et al. Computer Aided Numerical Methods for Hydrological Model Calibration: An Overview and Recent Development. Archives of Computational Methods in Engineering, $p$. $1-25,2017$.

KRUK, N. S. et al. Análise de Sensibilidade do Modelo Hidrológico Distribuído DHSVM aos Parâmetros de Vegetação. Revista Brasileira de Recursos Hídricos, v. 14, n. 1, p. 75-84, 2009.

LIU, Y. et al. A review on effectiveness of best management practices in improving hydrology and water quality: Needs and opportunities. Science of The Total Environment, v. 601-602, p. 580-593, 2017.

MELO NETO, J. DE O. et al. Simulação hidrológica escalar com o modelo SWAT. Revista Brasileira de Recursos Hídricos, v. 19, n. 1, p. 177-188, 2014.

MENDES, H. DE A. Metodologia para calibração do modelo hidrológico DHSVM. [s.I.] Universidade Federal do Espírito Santo, 2016.

MENDES, H. DE A.; CECÍLIO, R. A.; ZANETTI, S. S. Ferramenta para calibração automática do modelo hidrológico DHSVM. Engenharia na Agricultura, v. 25, p. 272-282, 2017.

MOLINA-NAVARRO, E. et al. The impact of the objective function in multi-site and multi-variable calibration of the SWAT model. Environmental Modelling \& Software, v. 93, p. 255-267, 2017.

MORIASI, D. N. et al. Model Evaluation Guidelines for Systematic Quantification of Accuracy in Watershed Simulations. Transactions of the ASABE, v. 50, n. 3, p. 885-900, 2007.

MORIASI, D. N. et al. Hydrologic and Water Quality Models: Performance Measures and Evaluation Criteria. Transactions of the ASABE, v. 58, n. 6, p. 1763-1785, 2015. 
NASH, J. E.; SUTCLIFFE, J. V. River flow forecasting through conceptual models part I - A discussion of principles. Journal of Hydrology, v. 10, n. 3, p. 282-290, 1970.

NGUYEN, H. H. et al. Modelling the impacts of altered management practices, land use and climate changes on the water quality of the Millbrook catchment-reservoir system in South Australia. Journal of Environmental Management, v. 202, p. 1-11, 2017.

NOORI, N.; KALIN, L. Coupling SWAT and ANN models for enhanced daily streamflow prediction. Journal of Hydrology, v. 533, p. 141-151, 2016.

OLIVEIRA, K. D. Modelagem hidrológica da bacia do rio Jucu utilizando o modelo DHSVM. [s.l.] Universidade Federal do Espírito Santo, 2014.

PINIEWSKI, M.; OKRUSZKO, T. Multi-Site Calibration and Validation of the Hydrological Component of SWAT in a Large Lowland Catchment. In: ŚWIĄTEK, D.; OKRUSZKO, T. (Eds.). . Modelling of Hydrological Processes in the Narew Catchment. Berlin: Springer, 2011. p. 1541.

PINTO, D. B. F. et al. Application of the Soil and Water Assessment Tool (SWAT) for sediment transport simulation at headwater watershed in Minas Gerais state, Brazil. Transactions of the ASABE, v. 56, n. 2, p. 697-709, 2013.

POTT, C. A.; FOHRER, N. Best management practices to reduce nitrate pollution in a rural watershed in Germany. Ambiente e Agua - An Interdisciplinary Journal of Applied Science, v. 12, n. 6, p. 888, 2017.

QI, C.; GRUNWALD, S. GIS-based hydrologic modeling in the Sandusky Watershed using SWAT. Transactions of the ASAE, v. 48, n. 1, p. 169-180, 2005.

RESHMA, T. et al. Optimization of Calibration Parameters for an Event Based Watershed Model Using Genetic Algorithm. Water Resources Management, v. 29, n. 13, p. 4589-4606, 7 out. 2015.

ROUHOLAHNEJAD, E. et al. A parallelization framework for calibration of hydrological models. Environmental Modelling and Software, v. 31, p. 28-36, 2012.

SANTOS, H. G. DOS et al. O novo mapa de solos do Brasil: legenda atualizada. Rio de Janeiro: EMBRAPA, 2011.

SHOURONG, W. et al. Numerical simulation experiments by nesting hydrology model DHSVM with regional climate model RegCM2 / China. Acta Meteorologica Sinica, v. 16, n. 4, p. 509518, 2002.

SHRESTHA, M. K. et al. Assessing SWAT models based on single and multi-site calibration for the simulation of flow and nutrient loads in the semi-arid Onkaparinga catchment in South Australia. Agricultural Water Management, v. 175, p. 61-71, 2016. 
SUMNER, N. R.; MICK FLEMING, P.; C. BATES, B. Calibration of a modified SFB model for twentyfive Australian catchments using simulated annealing. Journal of Hydrology, v. 197, n. 1-4, p. 166-188, 1997.

SUN, N. et al. A spatially distributed model for assessment of the effects of changing land use and climate on urban stream quality. Hydrological Processes, v. 30, n. 25, p. 4779-4798, 15 dez. 2016.

SURFLEET, C. G.; SKAUGSET, A. E.; MCDONNELL, J. J. Uncertainty assessment of forest road modeling with the Distributed Hydrology Soil Vegetation Model (DHSVM). Canadian Journal of Forest Research, v. 40, n. 7, p. 1397-1409, 2010.

THANAPAKPAWIN, P. et al. Effects of landuse change on the hydrologic regime of the Mae Chaem river basin, NW Thailand. Journal of Hydrology, v. 334, n. 1-2, p. 215-230, 2007.

TIGKAS, D.; CHRISTELIS, V.; TSAKIRIS, G. Comparative Study of Evolutionary Algorithms for the Automatic Calibration of the Medbasin-D Conceptual Hydrological Model. Environmental Processes, v. 3, n. 3, p. 629-644, 2016.

WANG, S. et al. Multi-site calibration, validation, and sensitivity analysis of the MIKE SHE Model for a large watershed in northern China. Hydrology and Earth System Sciences, v. 16, n. 12, p. 4621-4632, 2012.

WESTRICK, K. J.; STORCK, P.; MASS, C. F. Description and Evaluation of a Hydrometeorological Forecast System for Mountainous Watersheds. Weather and Forecasting, v. 17, n. 2, p. 250262, 2002.

WIGMOSTA, M. S. et al. The distributed hydrology soil vegetation model. In: SINGH, V. P.; FREVERT, D. K. (Eds.). . Mathematical models of small watershed hydrology and applications. Littleton: Water Resource Publications, 2002. p. 7-42.

WIGMOSTA, M. S.; VAIL, L. W.; LETTENMAIER, D. P. A distributed hydrology-vegetation model for complex terrain. Water Resources Research, v. 30, n. 6, p. 1665-1679, 1994.

WOLDESENBET, T. A. et al. Hydrological responses to land use/cover changes in the source region of the Upper Blue Nile Basin, Ethiopia. Science of The Total Environment, v. 575, p. 724-741, 2017.

YAO, C.; YANG, Z. Parameters optimization on DHSVM model based on a genetic algorithm. Frontiers of Earth Science in China, v. 3, n. 3, p. 374-380, 2009.

YEN, H. et al. Computational Procedure for Evaluating Sampling Techniques on Watershed Model Calibration. Journal of Hydrologic Engineering, v. 20, n. 7, p. 04014080, 2015a.

YEN, H. et al. Assessment of model predictions and parameter transferability by alternative land use data on watershed modeling. Journal of Hydrology, v. 527, p. 458-470, 2015b. 
ZHANG, L. et al. Comparison of baseline period choices for separating climate and land use/land cover change impacts on watershed hydrology using distributed hydrological models. Science of The Total Environment, v. 622-623, p. 1016-1028, 2018.

\section{Authorship contributions}

\section{1 - Roberto Avelino Cecílio}

Universidade Federal do Espírito Santo, Dourotado em Engenharia Agrícola https://orcid.org/0000-0003-2894-2481 - roberto.cecilio@ufes.br

Contribution: Conceptualization; Formal analysis; Funding acquisition; Supervision;

Writing - original draft

\section{2 - Helder de Amorim Mendes}

Universidade Federal do Espírito Santo, Doutorado em Ciências Florestais https://orcid.org/0000-0001-9648-9477 - helder.mendes@ufes.br

Contribution: Data curation; Formal analysis; Methodology; Software; Validation;

Writing - original draft

\section{3 - Sidney Sara Zanetti}

Universidade Federal do Espírito Santo, Doutorado em Produção Vegetal https://orcid.org/0000-0001-9135-3925 - sidney.zanetti@ufes.br

Contribution: Conceptualization; Formal analysis; Writing - original draft

\section{How to quote this article}

CECÍLIO, R. A.; MENDES, H. A.; ZANETTI, S. S. Multiple solutions, multi-site, and parameter transfer to calibrate DHSVM hydrological model. Ciência e Natura, Santa Maria, v. 43, e7, p. 1-32, 2021. Available from: https://doi.org/10.5902/2179460X42826. Accessed: Month Abbreviated. day, year. 